\title{
An Investigation of the Dispersion Forces in Weakly Bound Complexes Using Quantum Chemical and Multipole Expansion Methods
}

\author{
Wagner B. De Almeida* \\ Departamento de Química, ICEx, Universidade Federal de Minas Gerais, Campus Universitário, Pampulha, \\ 31270-901 Belo Horizonte - MG, Brazil
}

\begin{abstract}
Neste artigo a importância das forças de dispersão para a estabilização de dímeros moleculares fracamente ligados é investigada utilizando-se métodos quânticos ab initio de cálculo, além do tratamento pela Expansão de Multipolos para as forças de longo alcance. O efeito da correlação eletrônica na estrutura molecular e energias de ligação foram avaliados. Atenção é dada para a determinação das energias eletrostáticas e de dispersão através do cálculo ab initio de momentos de dipolo e quadrupolo elétricos e polarizabilidades. $\mathrm{O}$ cálculo dos coeficientes de dispersão $\mathrm{C}_{6}, \mathrm{C}_{8} \mathrm{e}$ $\mathrm{C}_{10}$ para a interação entre monômeros com simetria axial é discutido.
\end{abstract}

In this article the role of dispersion forces for the stabilization of weakly bound molecular dimers is investigated using quantum mechanical $a b$ initio methods and the Long-Range Multipole Expansion approach. The effect of the electron correlation on the molecular structure and binding energy is assessed. Attention is paid to the determination of the electrostatic and dispersion energies through the $a b$ initio calculated electric dipole and quadrupole moments and dipole polarizabilities. The calculation of the dispersion coefficients $\mathrm{C}_{6}, \mathrm{C}_{8}$ and $\mathrm{C}_{10}$ for the interaction of axial symmetric monomers is discussed.

Keywords: dispersion forces, multipole expansion, dimers, ab initio, electron correlation

\section{Introduction}

The theoretical calculation and experimental determination of intermolecular forces has been a fundamental subject discussed intensively on many review articles and books for many years. ${ }^{1-7}$ We can go back in time as far as 1965 to the meeting of the of the Faraday Society held at the University of Bristol on the $14^{\text {th }}, 15^{\text {th }}$ and $16^{\text {th }}$ September, published on the $40^{\text {th }}$ volume of the Discussion of the Faraday Society, ${ }^{1}$ and quote the lecture presented by Professor H.C. Longuet-Higgins and the summarizing remarks presented by Professor C.A. Coulson as outstanding reflections of the importance to intermolecular forces in chemistry. An issue of the Faraday Discussion of the Chemical Society published on 1982, dedicated to the subject of van der Waals molecules, deserves also to be mentioned. ${ }^{2}$ These two issues of the Discussions of the Faraday Society contains very interesting discussions on intermolecular forces and weakly bound molecular complexes, with the participation of leading theoretical and experimental scientists on this field.

\footnotetext{
* e-mail: wagner@netuno.qui.ufmg.br
}

Over the past 20 years there have been major advances in experimental methods, mainly spectroscopic ones, for observing gas phase weakly bound complexes along with outstanding progress in the theoretical methodologies available for describing intermolecular interactions in the gas phase. Nowadays, highly correlated ab initio methods can be used for the study of dimers constituted of small molecules. Within the Born-Oppenheimer approximation the potential energy surface (PES) can be investigated at the $a b$ initio level and therefore equilibrium and transition state structures can be located on the PES, and important phenomena such as tunneling motions can be investigated..$^{8-10}$ Nevertheless, more insight into the nature of the intermolecular interactions are attractively gained through the partition of the interaction energy into electrostatic, induction and dispersion contributions. ${ }^{3}$ Then the role played by the electronic correlation effects can be assessed based on the electrical properties of the free monomers, through the use of the short-range and longrange partition of the intermolecular potential, with the aid of the multipole expansion treatment. ${ }^{11}$

It is certainly of relevance to assess the performance of the theoretical quantum mechanical methods for 
describing the intermolecular interactions that take place in the gas phase, where the validity of the theoretical models developed to describe the gas phase experimental observations in the limit of the low temperature and pressure conditions can be confirmed. It is with these ideas in mind that this article was planned. Four dimeric species were selected and thought as representative of three main types of molecular complexes, true van der Waals dimer, $\left(\mathrm{H}_{2}\right)_{2}$, weakly bound complexes, $(\mathrm{HCCH})_{2}$, and hydrogen bonded dimers, $\left(\mathrm{H}_{2} \mathrm{O}\right)_{2}$ and $\left(\mathrm{HCN}_{2}\right)$, spanning T-shaped, Slipped-parallel, $\mathrm{C}_{2 \mathrm{v}}$-Trans-linear and Linear configurations. It is therefore believed that the results obtained here can be considered to encompass the most relevant class of molecular complexes. The aim of this work is to investigate the ability of the theoretical methods commonly used in the field of quantum chemistry to account for the dispersion forces present when molecules do interact to form a stable molecular complex.

\section{Methodology}

Figure 1 shows the intermolecular geometrical parameters with the definition of the intermolecular distance $\left(\mathrm{R}_{\mathrm{AB}}\right)$, angles $\left(\theta_{\mathrm{A}}\right.$ and $\left.\theta_{\mathrm{B}}\right)$ and dihedral angle $(\phi)$, with respect to the dipole moment vectors. The quantum chemical calculations were carried out with the intra and intermolecular geometrical parameters being fully optimized without symmetry or other geometrical constraint. For reason of simplicity only the intermolecular distances and angles will be presented here. Harmonic frequency calculations were also performed to characterize the located stationary point on the PES as minima (all frequencies are real) or first-order transition state (TS) structure (occurrence of one imaginary frequency). The Hartree-Fock (HF), ${ }^{12,14}$ Density Functional Theory (DFT) ${ }^{15}$ with the BLYP ${ }^{17-19}$ and PW91 ${ }^{20-24}$ exchange correlation functionals, and Moller-Plesset second order perturbation theory (MP2) 25,26 methods were used for geometry optimization and harmonic frequency calculations using the standard Pople's split valence basis set of triple zeta quality adding polarization and diffuse functions on all atoms $(6-311++G(2 d, 2 p)) .{ }^{27-34}$ Single point energy calculations were performed for the MP2 fully optimized geometry using the improved $6-311++\mathrm{G}(3 \mathrm{df}, 3 \mathrm{pd})$ basis set employing the MP4(SDTQ) treatment of the electronic correlation with single, double, triple and quadruple excitations. ${ }^{26}$ For the $\left(\mathrm{H}_{2}\right)_{2}$ van der Waals dimer the correlated consistent polarized valence triple-zeta and quadruple-zeta, augmented with diffuse functions on all atoms, (aug-cc-pVTZ and aug-cc-pVQZ) basis sets recent developed by Dunning and co-workers ${ }^{35-39}$ (see also
Davidson $^{40}$ ) were used for MP2 geometry optimization and frequency calculations, with single-point MP4(SDTQ) energy calculation being also performed. In addition, MP4(SDTQ) single-point energy calculation with the correlated consistent polarized valence quintuple-zeta basis set (aug-cc-pV5Z), for the MP2/aug-cc-pVQZ fully optimized geometry, was carried out, in order to assess the basis set limit behavior. The basis set superposition error (BSSE) was accounted for using the counterpoise method. ${ }^{41,42}$ For a more detailed discussion on the BSSE correction see van Duijneveldt et al. ${ }^{43}$ For a discussion on the effect of this correction for TS structures see Resende et al. . $^{4}$

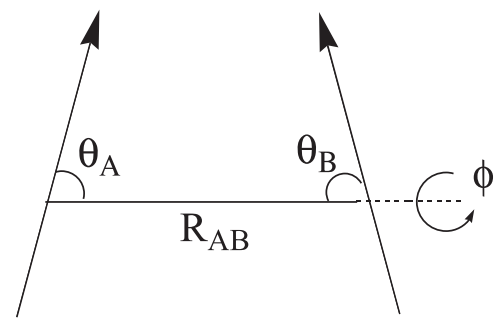

A

B

Figure 1. Geometric arrangement of two interacting monomers A and $\mathrm{B}$ as given by the dipole moment vectors. $\mathrm{R}_{\mathrm{AB}}$ is the centre of mass distance.

The spatial arrangements for the four dimers investigated in this work are depicted in Figure 2, along with the definition of the intermolecular geometrical parameters and dipole moment orientations. For the $\left(\mathrm{H}_{2}\right)_{2}$ and $(\mathrm{HCCH})_{2}$ dimers two structures, a true minimum (TShaped) and a first-order TS structure (Slipped-Parallel) were considered.

The multipole expansion for the molecular interaction energy, $\mathrm{U}(\mathrm{r})$, is given by the equation below. ${ }^{5-7}$

$\mathbf{U}=\sum_{n=k}^{\infty} U_{n} r^{-n}$

The coefficients for the interaction energy between two neutral molecules are given by equations (9-18). In these equations $\mu_{\mathrm{A}}, \mu_{\mathrm{B}}, \Theta_{\mathrm{A}}, \Theta_{\mathrm{B}}, \alpha_{\mathrm{A}}, \alpha_{\mathrm{B}}, \mathrm{E}_{\mathrm{A}}, \mathrm{E}_{\mathrm{B}}$ are respectively the permanent electric dipole moments, electric quadrupole moments, electric dipole polarizabilities and ionization energies of the monomers $\mathrm{A}$ and $\mathrm{B}$, with $\varepsilon_{0}$ being the vacuum permittivity. The temperature-dependent Boltzmann weighted average induction energy contribution, ${ }^{7}$ corresponding to dipole-dipole interactions for freely rotating molecules, is neglected.

The dipole moment vector, $\mu$ and quadrupole tensor, $\mathrm{Q}$, are given by ${ }^{45}$ 


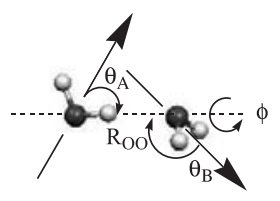

a)

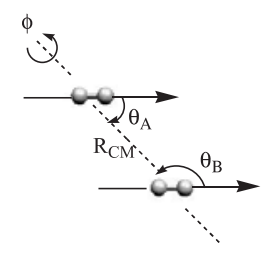

c)

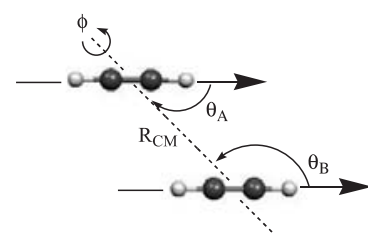

e)

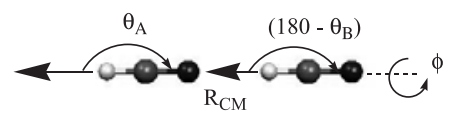

b)

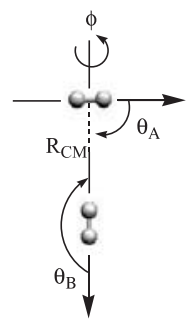

d)

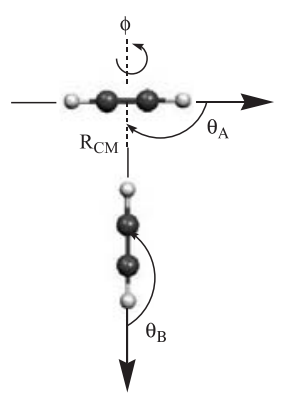

f)
Figure 2. Intermolecular geometrical parameters and spatial dipole orientation for the molecular complexes. (a) $\left(\mathrm{H}_{2} \mathrm{O}\right)_{2}$; (b) $(\mathrm{HCN})_{2}$; (c) $\left(\mathrm{H}_{2}\right)_{2}$ : Slipped-parallel; (d) $\left(\mathrm{H}_{2}\right)_{2}$ :T-Shaped; (e) $(\mathrm{HCCH})_{2}$ : Slippedparallel; (f) $(\mathrm{HCCH})_{2}: \mathrm{T}-$ Shaped

$\mu=\int \mathrm{r} \rho(\mathrm{r}) \mathrm{d} \tau$

$\mathrm{Q}=\int \operatorname{rr} \rho(\mathrm{r}) \mathrm{d} \tau$

where $\rho(\mathrm{r})$ is a continuous distribution of charge.

It is of common practice to define a different quadrupole moment tensor which is traceless, with only five independent elements,

$\Theta=1 / 2 \sum_{i} q_{i}\left(3 r_{i} r_{i}-r^{2} 1\right)$

where 1 is the unit tensor and the $1 / 2$ factor is conventional.

For axial symmetric and linear molecules the quadrupole tensor is diagonal in the molecular axes, with non-zero components $\Theta_{x x}=\Theta_{y y}=-1 / 2 \Theta_{z z}$. Then the quadrupole strength defined as $\Theta=\Theta_{z z}-\Theta_{x x}{ }_{x x}$ completely determines the traceless quadrupole moment tensor. The multipole expansion permit the electrostatic potential $(\mathrm{V}(\mathrm{r}))$ due to a charge distribution to be written in terms of the electric moments associated with the charge distribution. In the presence of an external electrostatic field, E, the energy of the charge distribution, W, can be written as ${ }^{46}$

$\mathrm{W}=\mathrm{qV}-\mu \cdot \mathrm{E}-1 / 3 \Theta: \mathrm{E}^{\prime}$

where $\mathrm{E}^{\prime}$ is the field gradient tensor containing terms like $\partial \mathrm{E}_{\mathrm{x}} / \partial_{\mathrm{y}}$.

The charge distribution can redistribute itself until its energy in the external field is minimized (polarization phenomenon), so the electric moments will change in the external field what can be studied by expanding the moments as a Taylor series. For the case of the electric dipole

$\mu(\mathrm{E})=\mu(\mathrm{E}=0)+\alpha \cdot \mathrm{E}+1 / 2 ! \beta^{\prime}: \mathrm{EE}+\ldots$

where the first term on the right side is the permanent electric dipole, the second term the induced dipole by the external field (given by the polarizability $\alpha$ ) and $\beta$ ' the first hyperpolarizability (similar expansions can also be written for higher electric multipoles, and so a quadrupole polarizability can be defined).

The dipole polarizability tensor $(\alpha)$ in principal axis is diagonal. For a molecule with symmetry the principal axes coincide with symmetry axes.

$\alpha=\left(\begin{array}{ccc}\alpha_{a a} & 0 & 0 \\ 0 & \alpha_{b b} & 0 \\ 0 & 0 & \alpha_{c c}\end{array}\right)$

For a linear molecule there is only two independent components denominated $\alpha_{\|}$and $\alpha_{\mathrm{A}}$, with the mean polarizability given by

$\bar{\alpha}=1 / 3\left(\alpha_{\|}+2 \alpha_{\mathrm{A}}\right)$

The long-range electrostatic, induction and dispersion interaction energy contributions are given below.

$$
\begin{aligned}
& \mathbf{U}_{\text {dipole-dipole }}=\frac{\mu_{A} \mu_{B}}{4 \pi \varepsilon_{0}} \frac{1}{R_{A B}^{3}}\left(2 \cos \theta_{A} \cos \theta_{B}-\operatorname{sen} \theta_{A} \operatorname{sen} \theta_{B} \cos \phi\right) \\
& \mathbf{U}_{\text {dipole-quadrupole }}=\frac{3}{2} \frac{1}{4 \pi \varepsilon_{0}} \frac{1}{R_{A B}^{4}}\left(\begin{array}{l}
\mu_{A} \Theta_{B}\left\{\cos \theta_{A}\left(3 \cos ^{2} \theta_{B}-1\right)+2 \sin \theta_{A} \sin \theta_{B} \cos \theta_{B} \cos \phi\right\}+ \\
\mu_{B} \Theta_{A}\left\{\cos \theta_{B}\left(3 \cos ^{2} \theta_{A}-1\right)+2 \operatorname{sen} \theta_{A} \operatorname{sen} \theta_{B} \cos \theta_{A} \cos \phi\right\}
\end{array}\right)
\end{aligned}
$$


$\mathbf{U}_{\text {quadrupole-quadrupole }}=\frac{3}{4} \frac{\Theta_{A} \Theta_{B}}{4 \pi \varepsilon_{0}} \frac{1}{R_{A B}^{5}}\left(\begin{array}{l}1-5 \cos ^{2} \theta_{A}-5 \cos ^{2} \theta_{B}+17 \cos ^{2} \theta_{A} \cos ^{2} \theta_{B} \\ +2 \operatorname{sen}^{2} \theta_{A} \operatorname{sen}^{2} \theta_{B} \cos ^{2} \phi \\ -16 \operatorname{sen} \theta_{A} \operatorname{sen} \theta_{B} \cos \theta_{A} \cos \theta_{B} \cos \phi\end{array}\right)$

$\mathbf{U}_{\text {induction }}=-\frac{1}{2} \frac{1}{4 \pi \varepsilon_{0}} \frac{1}{R_{A B}^{6}}\left(\alpha_{A} \mu_{B}^{2}\left(3 \cos ^{2} \theta_{B}+1\right)+\alpha_{B} \mu_{A}^{2}\left(3 \cos ^{2} \theta_{A}+1\right)\right)$

$\mathbf{U}_{\text {disp-R6 }}=-\frac{C_{6}}{R_{A B}^{6}}$

London Approximation: ${ }^{47}$

$\mathbf{C}_{6}=\frac{3}{2} \frac{\alpha_{A} \alpha_{B}}{\left(4 \pi \varepsilon_{0}\right)^{2}} \frac{E_{A} E_{B}}{\left(E_{A}+E_{B}\right)}$

In the quantum mechanical calculation of the energy of interaction between two molecules, the London dispersion forces appear in the second-order perturbation terms. They are called dispersion forces because the perturbation terms are expressed in terms of the same oscillator strengths, $f_{l k}$, as appear in the equations for the dispersion of light. ${ }^{5}$

The $\mathrm{C}_{8}$ and $\mathrm{C}_{10}$ dispersion coefficients were evaluated with the equations below. ${ }^{5,48,49}$

$$
\begin{aligned}
& \mathbf{U}_{\text {dipp-R } 8}=-\frac{C_{8}}{R_{A B}^{8}} \\
& \mathbf{U}_{\text {disp-r10 }}=-\frac{C_{10}}{R_{A B}^{10}} \\
& \mathbf{C}_{8}=\frac{45}{8} \frac{\alpha_{A} \alpha_{B}}{\left(4 \pi \varepsilon_{0}\right)^{2}} \frac{E_{A} E_{B}}{e^{2}}\left(\alpha_{A} \frac{E_{A}}{\left(2 E_{A}+E_{B}\right)}+\alpha_{B} \frac{E_{B}}{\left(E_{A}+2 E_{B}\right)}\right) \\
& \mathbf{C}_{10}=\frac{315}{16} \frac{\alpha_{A}^{2} \alpha_{B}^{2}}{\left(4 \pi \varepsilon_{0}\right)^{2}}\left(\frac{E_{A}^{2} E_{B}^{2}}{e^{4}\left(E_{A}+E_{B}\right)}\right)
\end{aligned}
$$

The dispersion forces between asymmetric molecules at large separations can be calculated with equations (19) and (20), which takes into account the effect of the spatial orientation of the dipoles not present in the simple London approximation which is valid for spherically symmetrical systems. A deviation from isotropicity of the polarizability is considered and the magnitude of the dispersion energy will depend on the mutual orientation of the two interacting molecules. ${ }^{5}$

$$
\mathbf{U}_{\text {disp-R6-asym }}=-\frac{C_{6}^{\text {asym }}}{R_{A B}^{6}}
$$

All quantum chemical calculations were done using the GAUSSIAN 94/98 ab initio package ${ }^{50}$ as implemented on Digital/alpha-500au, SilicomGraphics R14000 workstations and Microcomputer Pentium-4, LYNUX Operating System, at the Laboratório de Química Computacional e Modelagem Molecular (LQC-MM), Departamento de Química, UFMG. The multipole expansion calculations for the molecular interaction energies were carried out using a Fortran code developed in the LQC-MM.

\section{Results and Discussion}

The results reported in the next Tables include DFT calculations using only two functionals BLYP and PW91. The aim here is not a detailed investigation of the performance of exchange-correlation functionals for describing molecular complexes. This subject has already been addressed in the literature and the difficulty of the DFT method for treating weakly bound dimers is well known. The interest here is mainly to assess how much electronic correlation these functionals contain, the BSSE effects, and also to confirm their ability to reproduce the interaction energy of H-bonded dimers, due to the plausibility of using DFT methods in larger molecular complexes where hydrogen bonding plays an important role. The reason for choosing the BLYP and PW91 functionals is our previous experience with the (PCCP) weakly bound complex study ${ }^{51}$ where various distinct functionals were employed, using basis sets of different sizes. The results obtained could be grouped into two

$$
\begin{aligned}
& \mathbf{C}_{6}{ }^{\text {asym }}=\frac{1}{\left(4 \pi \varepsilon_{0}\right)^{2}}\left[\begin{array}{l}
\left(A-B-B^{\prime}+C\right)\left\{\sin \theta_{A} \sin \theta_{B} \cos \phi+2 \cos \theta_{A} \cos \theta_{B}\right\}^{2}+ \\
3(B-C) \cos ^{2} \theta_{A}+3\left(B^{\prime}-C\right) \cos ^{2} \theta_{B}+\left(B+B^{\prime}+4 C\right)
\end{array}\right] \\
& A=\frac{\alpha_{\|}^{A} \alpha_{\|}^{B}}{4} \frac{E_{A} E_{B}}{\left(E_{A}+E_{B}\right)} ; B=\frac{\alpha_{\|}^{A} \alpha_{\perp}^{B}}{4} \frac{E_{A} E_{B}}{\left(E_{A}+E_{B}\right)} ; B^{\prime}=\frac{\alpha_{\perp}^{A} \alpha_{\|}^{B}}{4} \frac{E_{A} E_{B}}{\left(E_{A}+E_{B}\right)} ; C=\frac{\alpha_{\perp}^{A} \alpha_{\perp}^{B}}{4} \frac{E_{A} E_{B}}{\left(E_{A}+E_{B}\right)}
\end{aligned}
$$


classes, and these two functionals were representatives of the results obtained among various exchange-correlation functionals used. The PW91 is the only functional that predicted a bound (PCCP) ${ }_{2}$ dimer, in agreement with PostHF calculations. The other functionals employed, including BLYP, yielded an unbound dimer. So, a comparison between BLYP and PW91, for the description of the weakly bound, van der Waals and H-bonded dimers studied here may be interesting. In addition, we have recently used the BLYP/6-31G(d,p) level of calculation for the investigation of the hydration of $\alpha$-cyclodextrin $(\alpha-C D)^{52}$ and the agreement with experiment for enthalpy and Gibbs free energy of hydration was very good. Therefore, it is opportune to assess the behavior of the BLYP functional for the evaluation of interaction energies compared with Post-HF calculations, since DFT single point energy calculations can be performed at an affordable computational cost for large molecular complexes, such as $\alpha$-CD dimer. ${ }^{53}$

The calculated structural and energetic data for the water dimer is given in Table 1, where the experimental parameters are also quoted. As it has already been reported in the literature the agreement with experiment is fairly good. The HF energies are underestimated relative to the Post-HF ones due to the lack of electronic correlation. The BLYP energies are underestimated while the PW91 ones are overestimated compared to the MP4(SDTQ) values, within the same basis set. The BSSE values are much smaller at the HF and DFT levels, showing that improved basis sets are required in Post-HF calculations. With the standard Pople's 6-311++G(3df,3pd) basis set the BSSE correction is $14 \%$ of the total MP4(SDTQ) interaction energy, still a sizeable value. An improved value for the water dimer can be obtained using the correlated consistent polarized valence quadruple-zeta, augmented with diffuse functions on all atoms basis set (aug-cc-pVQZ) both at the MP4(SDTQ) and coupled cluster with single, double and non-iterative triple excitations $(\mathrm{CCSD}-\mathrm{T})^{56}$ levels of calculation, which yield a value for the BSSE of $0.14 \mathrm{kcal}$ mol $^{-1}$, less than $3 \%$ of the ab initio stabilization energy. ${ }^{57}$ In addition the MP4(SDTQ)/aug-cc-pVQZ value of -4.94 kcal $\mathrm{mol}^{-1}$ value from De Almeida ${ }^{57}$ is closer to the experimental value of $-4.78 \mathrm{kcal} \mathrm{mol}^{-1}$ than the one reported here with the $6-311++\mathrm{G}(3 \mathrm{df}, 3 \mathrm{pd})$ basis set (the error is $3 \%$ ), confirming the importance of the use of a good quality basis set in Post-HF stabilization energy calculations. In can be seen from the results of De Almeida ${ }^{57}$ that a practically BSSE free value would be attained using the quintuple-zeta basis set ((aug-cc-pV5Z). It will be shown later in this article that this is indeed the case for the $\left(\mathrm{H}_{2}\right)_{2}$ van der Waals dimer. It can be seen from Table 1 that, after the BSSE correction is accounted for, the BLYP energies agree better with the MP4(SDTQ) values than the PW91 ones, providing an indication that the PW91 energy

Table 1. Geometrical parameters and interaction energy values for the $\left(\mathrm{H}_{2} \mathrm{O}\right)_{2}$ dimer. Distances in angstrom, angles in degrees and energies in units of $\mathrm{kcal} \mathrm{mol}^{-1}$

\begin{tabular}{|c|c|c|c|c|c|c|}
\hline $\begin{array}{l}\mathrm{H}_{2} \mathrm{O} \\
\text { Dimer }\end{array}$ & $\begin{array}{l}\mathrm{HF} / 6-311++\mathrm{G} \\
(2 \mathrm{~d}, 2 \mathrm{p})\end{array}$ & $\begin{array}{l}\mathrm{HF} / 6-311++\mathrm{G} \\
(3 \mathrm{df}, 3 \mathrm{pd}) / /^{\mathrm{d}}\end{array}$ & $\begin{array}{l}\text { BLYP/6-311++G } \\
(2 \mathrm{~d}, 2 \mathrm{p})\end{array}$ & $\begin{array}{l}\text { BLYP/6-311++G } \\
(3 \mathrm{df}, 3 \mathrm{pd}) / /{ }^{\mathrm{d}}\end{array}$ & $\begin{array}{l}\text { PW91/6-311++G } \\
(2 \mathrm{~d}, 2 \mathrm{p})\end{array}$ & $\begin{array}{l}\text { PW91/6-311++G } \\
(3 \mathrm{df}, 3 \mathrm{pd}) / /^{\mathrm{d}}\end{array}$ \\
\hline $\mathrm{R}_{\mathrm{oO}}$ & 3.035 & - & 2.946 & - & 2.878 & - \\
\hline$\theta_{\mathrm{A}}$ & 56.5 & - & 57.8 & - & 58.4 & - \\
\hline$\theta_{\mathrm{B}}$ & 130.8 & - & 119.1 & - & 115.3 & - \\
\hline$\phi$ & 180.0 & - & 179.1 & - & 179.1 & - \\
\hline $\mathrm{w}_{1}{ }^{\mathrm{h}}$ & 126 & - & 129 & - & 141 & - \\
\hline$\Delta \mathrm{E}_{\text {ele-nuc }}$ & -4.07 & -3.91 & -4.60 & -4.45 & -5.93 & -5.81 \\
\hline$\Delta \mathrm{E}^{\mathrm{BSSE}}$ & $0.26(6 \%)^{f}$ & $0.20(5 \%)^{\mathrm{f}}$ & $0.35(8 \%)^{\mathrm{f}}$ & $0.27(6 \%)^{f}$ & $0.42(7 \%)^{f}$ & $0.38(7 \%)^{f}$ \\
\hline$\Delta \mathrm{E}^{\mathrm{BSSEc}}$ & -3.82 & -3.71 & -4.25 & -4.18 & -5.51 & -5.43 \\
\hline $\begin{array}{l}\mathrm{H}_{2} \mathrm{O} \\
\text { Dimer }\end{array}$ & $\begin{array}{l}\text { MP2/6-311++G } \\
(2 \mathrm{~d}, 2 \mathrm{p})\end{array}$ & $\begin{array}{l}\mathrm{MP} 4(\mathrm{SDQ}) / 6-311++\mathrm{G} \\
(2 \mathrm{~d}, 2 \mathrm{p}) / \mathrm{e}^{\mathrm{e}}\end{array}$ & $\begin{array}{l}\text { MP4(SDTQ)/6-311++G } \\
(2 \mathrm{~d}, 2 \mathrm{p}) / / \mathrm{e}^{\mathrm{e}}\end{array}$ & $\begin{array}{l}\text { MP2/6-311++G } \\
(3 \mathrm{df}, 3 \mathrm{pd}) / /^{\mathrm{e}}\end{array}$ & $\begin{array}{l}\mathrm{MP} 4(\mathrm{SDQ}) / 6-311++\mathrm{G} \\
(3 \mathrm{df}, 3 \mathrm{pd}) / /^{\mathrm{e}}\end{array}$ & $\begin{array}{l}\text { MP4(SDTQ)/6-311++G } \\
(3 \mathrm{df}, 3 \mathrm{pd}) / / \mathrm{e}^{\mathrm{e}}\end{array}$ \\
\hline $\mathrm{R}_{\mathrm{OO}}$ & $2.919[2.98 \pm 0.01]^{\mathrm{a}}$ & - & - & $(2.903)^{\mathrm{b}}$ & - & - \\
\hline$\theta_{\mathrm{A}}$ & $56.8[51 \pm 6]^{\mathrm{a}}$ & - & - & $(56.5)^{\mathrm{b}}$ & - & - \\
\hline$\theta_{\mathrm{B}}$ & $123.7[122 \pm 6]^{\mathrm{a}}$ & - & - & $(125.8)^{b}$ & - & - \\
\hline$\phi$ & 180.0 & - & - & & - & - \\
\hline $\mathrm{w}_{1}{ }^{\mathrm{h}}$ & 134 & - & - & - & - & - \\
\hline$\Delta \mathrm{E}_{\text {ele-nuc }}$ & $-5.36(-3.89)^{c}$ & -5.09 & -5.36 & $-5.30(-3.82)^{c}$ & -5.06 & -5.33 \\
\hline$\Delta \mathrm{E}^{\mathrm{BSSE}}$ & $0.87(16 \%)^{f}$ & $0.82(16 \%)^{\mathrm{f}}$ & $0.96(18 \%)^{f}$ & $0.68(13 \%)^{f}$ & $0.65(13 \%)^{f}$ & $0.74(14 \%)^{\mathrm{f}}$ \\
\hline$\Delta \mathrm{E}^{\mathrm{BSSEC}}$ & -4.49 & -4.27 & -4.40 & -4.62 & -4.41 & $-4.59[-4.78]^{\mathrm{g}}$ \\
\hline
\end{tabular}

${ }^{a}$ Experimental value from refs. ${ }^{54,55}$; ${ }^{\mathrm{b}} \mathrm{MP} 2 / 6-311++\mathrm{G}(3 \mathrm{df}, 3 \mathrm{pd})$ fully optimized value; ${ }^{\mathrm{c}}$ Hartree-Fock contribution to the MP2 energy; ${ }^{\mathrm{d}}$ The double slash indicates that a single point calculation at the geometry optimized with the $6-311++\mathrm{G}(2 \mathrm{~d}, 2 \mathrm{p})$ basis set was performed; ${ }^{\text {e The double }}$ slash indicates that a single point energy calculation at the fully optimized MP $2 / 6-311++G(2 d, 2 p)$ geometry was performed; ${ }^{f}$ Percentage value relative to the $\Delta \mathrm{E}_{\text {ele-nuc }} \cdot\left(=\left(\Delta \mathrm{E}^{\mathrm{BSSE}} / \Delta \mathrm{E}_{\text {ele-nuc }}\right) \times 100\right) ;{ }^{\mathrm{g}}$ Experimental value from refs. ${ }^{58,54,55} ;{ }^{\mathrm{h}} \mathrm{W}_{1}$ is the lowest harmonic frequency (in units of $\left.\mathrm{cm}{ }^{-1}\right)$ which characterize the located stationary point as true minima or first-order TS structure. 
Table 2. Geometrical parameters and interaction energy values for the $(\mathrm{HCN})_{2}$ linear dimer. Distances in angstrom, angles in degrees and energies in units of $\mathrm{kcal} \mathrm{mol}^{-1}$

\begin{tabular}{|c|c|c|c|c|c|c|}
\hline $\begin{array}{l}\text { HCN } \\
\text { Dimer }\end{array}$ & $\begin{array}{l}\mathrm{HF} / 6-311++\mathrm{G} \\
(2 \mathrm{~d}, 2 \mathrm{p})\end{array}$ & $\begin{array}{l}\mathrm{HF} / 6-311++\mathrm{G} \\
(3 \mathrm{df}, 3 \mathrm{pd}) / / \mathrm{e}^{\mathrm{e}}\end{array}$ & $\begin{array}{l}\text { BLYP/6-311++G } \\
(2 \mathrm{~d}, 2 \mathrm{p})\end{array}$ & $\begin{array}{l}\text { BLYP/6-311++G } \\
(3 \mathrm{df}, 3 \mathrm{pd}) / / \mathrm{e}^{\mathrm{e}}\end{array}$ & $\begin{array}{l}\text { PW91/6-311++G } \\
(2 \mathrm{~d}, 2 \mathrm{p})\end{array}$ & $\begin{array}{l}\text { PW91/6-311++G } \\
(3 \mathrm{df}, 3 \mathrm{pd}) / / \mathrm{e}^{\mathrm{a}}\end{array}$ \\
\hline$R_{C M}$ & 4.530 & - & 4.496 & - & 4.422 & - \\
\hline$\theta_{\mathrm{A}}$ & 180.0 & - & 180.0 & - & 180.0 & - \\
\hline$\theta_{\mathrm{B}}$ & 0.0 & - & 0.0 & - & 0.0 & - \\
\hline$\phi$ & 0.0 & - & 0.0 & - & 0.0 & - \\
\hline $\mathrm{w}_{1}{ }^{\mathrm{g}}$ & 49.0 & - & 48.4 & - & 46.4 & - \\
\hline$\Delta \mathrm{E}_{\text {ele-nuc }}$ & -4.23 & -4.27 & -3.79 & -3.60 & -4.80 & -4.66 \\
\hline$\Delta E^{\text {BSSE }}$ & $0.063(2 \%)^{\mathrm{a}}$ & $0.13(3 \%)^{\mathrm{a}}$ & -0.11 & -0.12 & 0.023 & -0.05 \\
\hline$\Delta E^{\text {BSSEC }}$ & -4.17 & -4.14 & - & - & -4.78 & - \\
\hline $\begin{array}{l}\mathrm{HCN} \\
\text { Dimer }\end{array}$ & $\begin{array}{l}\text { MP2/6-311++G } \\
(2 d, 2 p)\end{array}$ & $\begin{array}{l}\mathrm{MP} 4(\mathrm{SDQ}) / 6-311++\mathrm{G} \\
(2 \mathrm{~d}, 2 \mathrm{p}) / /^{\mathrm{f}}\end{array}$ & $\begin{array}{l}\mathrm{MP} 4(\mathrm{SDTQ}) / 6-311++\mathrm{G} \\
(2 \mathrm{~d}, 2 \mathrm{p}) / /^{\mathrm{f}}\end{array}$ & $\begin{array}{l}\mathrm{MP} 2 / 6-311++\mathrm{G} \\
(3 \mathrm{df}, 3 \mathrm{pd}) / /^{\mathrm{f}}\end{array}$ & $\begin{array}{l}\mathrm{MP} 4(\mathrm{SDQ}) / 6-311++\mathrm{G} \\
(3 \mathrm{df}, 3 \mathrm{pd}) / /^{\mathrm{f}}\end{array}$ & $\begin{array}{l}\mathrm{MP} 4(\mathrm{SDTQ}) / 6-311++\mathrm{G} \\
(3 \mathrm{df}, 3 \mathrm{pd}) / /^{\mathrm{f}}\end{array}$ \\
\hline$R_{\mathrm{OM}}$ & $4.441[4.382]^{\mathrm{b}}$ & & & & & \\
\hline$\theta_{\mathrm{A}}$ & 180.0 & & & & & \\
\hline$\theta_{\mathrm{B}}$ & 0.0 & & & & & \\
\hline$\phi$ & 0.0 & & & & & \\
\hline $\mathrm{w}_{1}{ }^{\mathrm{g}}$ & 52.5 & & & & & \\
\hline$\Delta \mathrm{E}_{\text {ele-nuc }}$ & $-5.04(-4.21)^{c}$ & -4.80 & -4.93 & $-5.15(-4.25)^{\mathrm{c}}$ & -4.91 & -5.06 \\
\hline$\Delta \mathrm{E}^{\mathrm{BSSE}}$ & $0.58(12 \%)^{\mathrm{a}}$ & $0.61(13 \%)^{\mathrm{a}}$ & $0.63(13 \%)^{\mathrm{a}}$ & $0.55(11 \%)^{\mathrm{a}}$ & $0.55(11 \%)^{\mathrm{a}}$ & $0.58(11 \%)^{\mathrm{a}}$ \\
\hline$\Delta E^{\text {BSSEC }}$ & -4.46 & -4.19 & -4.30 & -4.60 & -4.36 & $-4.48[-4.40]^{\mathrm{d}}$ \\
\hline
\end{tabular}

${ }^{a}$ Percentage value relative to the $\Delta \mathrm{E}_{\text {ele-nuc }}\left(=\left(\Delta \mathrm{E}^{\mathrm{BSSE}} / \Delta \mathrm{E}_{\text {ele-nuc }}\right) \times 100\right) ;{ }^{\mathrm{b}}$ Experimental value from ref. ${ }^{59}$; ${ }^{\mathrm{c}}$ Hartree-Fock contribution to the MP2 energy; ${ }^{d}$ Experimental value from ref. ${ }^{60}$; ${ }^{e}$ The double slash indicates that a single point calculation at the geometry optimized with the 6$311++\mathrm{G}(2 \mathrm{~d}, 2 \mathrm{p})$ basis set was performed; ${ }^{\mathrm{f}}$ The double slash indicates that a single point energy calculation at the fully optimized MP2/6$311++\mathrm{G}(2 \mathrm{~d}, 2 \mathrm{p})$ geometry was performed; ${ }^{\mathrm{g}} \mathrm{W}_{1}$ is the lowest harmonic frequency (in units of $\mathrm{cm}^{-1}$ ) which characterize the located stationary point as true minima or first-order TS structure.

values may be somewhat exaggerated for the $\left(\mathrm{H}_{2} \mathrm{O}\right)_{2}$ dimer and, possibly, water clusters.

Table 2 show the results obtained for the linear $(\mathrm{HCN})_{2}$ dimer. The strength of this hydrogen bonded dimer is very similar to the water dimer, with the $a b$ initio BSSE being virtually the same. The experimentally observed intermolecular distance is in fair agreement with the MP2 value. It can be noted that the BSSE correction for the DFT calculations tend to be negative or very small, different from the water dimer case where they are small but larger than the ab initio HF values. This BSSE behavior for the DFT calculations have been observed for the $\left(\mathrm{H}_{2}\right)_{2}$ and $(\mathrm{HCCH})_{2}$ dimers and it appears that the counterpoise method may not be appropriate in DFT calculations of molecular complexes, where the stabilization energy trend with the basis set size does not follow the same pattern as $a b$ initio $\mathrm{HF}$ and Post-HF energy calculations. So, it seems that there is no meaning in correcting DFT stabilization energies for BSSE. However, the results reported here may not be conclusive and a more detailed study is necessary. Therefore, BSSE uncorrected DFT stabilization energies is more advisable to be used. Another comparison with the water dimer reveals that the PW91 energies for the HCN dimer are larger than the BLYP ones but underestimated compared to the MP4(SDTQ) BSSE uncorrected values. So from the results of Tables 1 and 2, it can not be concluded that the PW91 functional systematically predicts overestimated stabilization energies compared to $a b$ initio Post-HF results for H-bonded dimers, where electrostatic interactions tend to play a major role. However, the BLYP energies follow a regular pattern for both hydrogen bonded dimers being systematically smaller than the corresponding Post-HF values but satisfactorily close, particularly for the water dimer where the deviation is ca. $3 \%$ (calculated in relation to the ab initio BSSE corrected value).

The results for the acetylene dimer are given in Table 3. For this weakly bound complex the electron correlation effects becomes very important, with a competition between electrostatic and dispersion effects promptly seen. The T-Shaped dimer is the global minimum on the PES, however there is a small barrier for interconversion between two equivalent T-Shaped forms through a Slipped-parallel TS structure, with the consequent splitting in the rotational spectrum being experimentally observed. ${ }^{61}$ The experimental geometry is very satisfactorily reproduced a the MP2 level of theory. It can be seen that the BLYP functional is inappropriate to describe this weakly bound dimer, with the stabilization energy being far less than the HF value. In this case the correlation contribution to the stabilization energy is really substantial with the T-shaped being stabilized by $c a .60 \%$ in relation to the HF prediction 
Table 3. Geometrical parameters and interaction energy values for the ( $\mathrm{HCCH})_{2}$ T-Shaped and Slipped-Parallel dimers. Distances in angstrom, angles in degrees and energies in units of kcal mol${ }^{-1}$

\begin{tabular}{|c|c|c|c|c|c|c|}
\hline $\begin{array}{l}(\mathrm{HCCH})_{2} \\
\text { T-Shaped }\end{array}$ & $\begin{array}{l}\mathrm{HF} / 6-311++\mathrm{G} \\
(2 \mathrm{~d}, 2 \mathrm{p})\end{array}$ & $\begin{array}{l}\mathrm{HF} / 6-311++\mathrm{G} \\
(3 \mathrm{df}, 3 \mathrm{pd})\end{array}$ & $\begin{array}{l}\text { BLYP/6-311++G } \\
(2 \mathrm{~d}, 2 \mathrm{p})\end{array}$ & $\begin{array}{l}\text { BLYP/6-311++G } \\
\text { (3df,3pd) }\end{array}$ & $\begin{array}{l}\text { PW91/6-311++G } \\
(2 d, 2 p)\end{array}$ & $\begin{array}{l}\text { PW91/6-311++G } \\
\text { (3df,3pd) }\end{array}$ \\
\hline $\mathrm{R}_{\mathrm{CM}}$ & 4.721 & - & 4.617 & - & 4.389 & - \\
\hline$\theta_{\mathrm{A}}$ & 87.8 & - & 87.8 & - & 87.8 & - \\
\hline$\theta_{\mathrm{B}}$ & 179.5 & - & 179.2 & - & 178.8 & - \\
\hline$\phi$ & 0.0 & - & 0.0 & - & 0.0 & - \\
\hline $\mathrm{w}_{1}{ }^{\mathrm{g}}$ & 24.9 & - & 29.0 & - & 36.5 & - \\
\hline$\Delta \mathrm{E}_{\text {ele-nuc }}$ & -0.75 & -0.92 & -0.56 & -0.46 & -1.52 & -1.39 \\
\hline 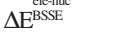 & -0.032 & $0.14(15 \%)^{\mathrm{a}}$ & $0.0033(0.6)^{\mathrm{a}}$ & -0.066 & $0.0405(3 \%)^{\mathrm{a}}$ & $0.092(7 \%)^{\mathrm{a}}$ \\
\hline$\Delta \mathrm{E}^{\mathrm{BSSEc}}$ & - & -0.78 & -0.56 & - & -1.48 & -1.30 \\
\hline $\begin{array}{l}(\mathrm{HCCH})_{2} \\
\text { T-Shaped }\end{array}$ & $\begin{array}{l}\text { MP2/6-311++G } \\
(2 \mathrm{~d}, 2 \mathrm{p})\end{array}$ & $\begin{array}{l}\text { MP4(SDQ)/6-311++G } \\
(2 \mathrm{~d}, 2 \mathrm{p})\end{array}$ & $\begin{array}{l}\text { MP4(SDTQ)/6-311++G } \\
(2 \mathrm{~d}, 2 \mathrm{p})\end{array}$ & $\begin{array}{l}\text { MP2/6-311++G } \\
(3 \mathrm{df}, 3 \mathrm{pd})\end{array}$ & $\begin{array}{l}\text { MP4(SDQ)/6-311++G } \\
\text { (3df,3pd) }\end{array}$ & $\begin{array}{l}\text { MP4(SDTQ)/6-311++G } \\
(3 \mathrm{df}, 3 \mathrm{pd})\end{array}$ \\
\hline $\mathrm{R}_{\mathrm{CM}}$ & $4.346[4.38]^{\mathrm{b}}$ & & & & & \\
\hline$\theta_{\mathrm{A}}$ & 87.8 & & & & & \\
\hline$\theta_{\mathrm{B}}$ & 178.7 & & & & & \\
\hline$\phi$ & 0.0 & & & & & \\
\hline $\mathrm{w}_{1}^{\mathrm{g}}$ & 35.5 & & & & & \\
\hline$\Delta \mathrm{E}_{\text {ele-nuc }}$ & $-1.80(-0.39)^{\mathrm{c}}$ & -1.47 & -1.71 & $-1.87(-0.27)^{c}$ & -1.46 & -1.76 \\
\hline \multirow[t]{3}{*}{$\Delta \mathrm{E}^{\text {ele-sSEC }}$} & $0.48(27 \%)$ & $0.43(29 \%)^{\mathrm{a}}$ & $0.53(31 \%)^{\mathrm{a}}$ & $0.44(23 \%)^{\mathrm{a}}$ & $0.35(24 \%)^{\mathrm{a}}$ & $0.44(25 \%)^{\mathrm{a}}$ \\
\hline & $(-0.074)^{c}$ & & & $(-0.058)^{c}$ & & \\
\hline & $(19 \%)^{\mathrm{c}}$ & & & $(22 \%)^{\mathrm{c}}$ & & \\
\hline$\Delta \mathrm{E}^{\mathrm{BSSEc}}$ & -1.32 & -1.04 & -1.18 & -1.43 & -1.11 & $-1.32[-1.09]^{\mathrm{d}}$ \\
\hline $\begin{array}{l}(\mathrm{HCCH})_{2} \\
\text { Slipp-Par }\end{array}$ & $\begin{array}{l}\mathrm{HF} / 6-311++\mathrm{G} \\
(2 \mathrm{~d}, 2 \mathrm{p})\end{array}$ & $\begin{array}{l}\mathrm{HF} / 6-311++\mathrm{G} \\
(3 \mathrm{df}, 3 \mathrm{pd})\end{array}$ & $\begin{array}{l}\text { BLYP/6-311++G } \\
(2 \mathrm{~d}, 2 \mathrm{p})\end{array}$ & $\begin{array}{l}\text { BLYP/6-311++G } \\
\text { (3df,3pd) }\end{array}$ & $\begin{array}{l}\text { PW91/6-311++G } \\
(2 \mathrm{~d}, 2 \mathrm{p})\end{array}$ & $\begin{array}{l}\text { PW91/6-311++G } \\
\text { (3df,3pd) }\end{array}$ \\
\hline $\mathrm{R}_{\mathrm{CM}}$ & 4.637 & - & 4.753 & - & 4.338 & - \\
\hline$\theta_{\mathrm{A}}$ & 42.4 & - & 41.7 & - & 41.7 & - \\
\hline$\theta_{\mathrm{B}}$ & 135.9 & - & 136.7 & - & 136.2 & - \\
\hline$\phi$ & 0.0 & - & 0.0 & - & 0.0 & - \\
\hline $\mathrm{w}_{1}^{\mathrm{g}}$ & $20.2 \mathrm{i}$ & - & $26.8 \mathrm{i}$ & - & 27.0i & - \\
\hline$\Delta \mathrm{E}_{\text {ele-nuc }}$ & -0.60 & -0.75 & -0.31 & -0.22 & -1.22 & -1.09 \\
\hline$\Delta \mathrm{E}^{\mathrm{e} B S \mathrm{BSE}}$ & -0.13 & $0.024(3 \%)^{\mathrm{a}}$ & -0.045 & -0.10 & $0.032(3 \%)^{\mathrm{a}}$ & $0.046(4 \%)^{\mathrm{a}}$ \\
\hline$\Delta \mathrm{E}^{\mathrm{BSSEc}}$ & - & -0.73 & - & - & -1.19 & -1.05 \\
\hline $\begin{array}{l}(\mathrm{HCCH})_{2} \\
\text { Slipp-Par }\end{array}$ & $\begin{array}{l}\text { MP2/6-311++G } \\
(2 \mathrm{~d}, 2 \mathrm{p})\end{array}$ & $\begin{array}{l}\text { MP4(SDQ)/6-311++G } \\
(2 \mathrm{~d}, 2 \mathrm{p})\end{array}$ & $\begin{array}{l}\text { MP4(SDTQ)/6-311++G } \\
(2 \mathrm{~d}, 2 \mathrm{p})\end{array}$ & $\begin{array}{l}\text { MP2/6-311++G } \\
(3 \mathrm{df}, 3 \mathrm{pd})\end{array}$ & $\begin{array}{l}\text { MP4(SDQ)/6-311++G } \\
\text { (3df,3pd) }\end{array}$ & $\begin{array}{l}\text { MP4(SDTQ)/6-311++G } \\
(3 \mathrm{df}, 3 \mathrm{pd})\end{array}$ \\
\hline $\mathrm{R}_{\mathrm{CM}}$ & $4.212[4.44]^{\mathrm{b}}$ & & & & & \\
\hline$\theta_{\mathrm{A}}$ & $41.7[46.1]^{\mathrm{b}}$ & & & & & \\
\hline$\theta_{\mathrm{B}}$ & 136.1 [133.9] $^{\mathrm{b}}$ & & & & & \\
\hline$\phi$ & 0.0 & & & & & \\
\hline $\mathrm{w}_{1}{ }^{\mathrm{g}}$ & $22.8 \mathrm{i}$ & & & & & \\
\hline$\Delta \mathrm{E}_{\text {ele-nuc }}$ & $-1.52(-0.23)^{\mathrm{c}}$ & -1.17 & -1.40 & $-1.63(-0.17)^{\mathrm{c}}$ & -1.24 & -1.51 \\
\hline$\Delta \mathrm{E}^{\mathrm{BSSE}}$ & $0.32(21 \%)^{\mathrm{a}}$ & $0.27(23 \%)^{\mathrm{a}}$ & $0.35(25 \%)^{\mathrm{a}}$ & $0.29(18 \%)^{\mathrm{a}}$ & $0.23(19 \%)^{a}$ & $0.30(20 \%)^{\mathrm{a}}$ \\
\hline & $(-0.083)^{c}$ & & & $(-0.086)^{c}$ & & \\
\hline & $(36 \%)^{\mathrm{c}}$ & & & $(50 \%)^{c}$ & & \\
\hline$\Delta \mathrm{E}^{\mathrm{BSSEC}}$ & $-1.20\left(42.0 \mathrm{~cm}^{-1}\right)^{\mathrm{e}}$ & $-0.90\left(49.0 \mathrm{~cm}^{-1}\right)^{\mathrm{e}}$ & $-1.05\left(45.5 \mathrm{~cm}^{-1}\right)^{\mathrm{e}}$ & $-1.34\left(31.5 \mathrm{~cm}^{-1}\right)^{\mathrm{e}}$ & $-1.01\left(35.0 \mathrm{~cm}^{-1}\right)^{\mathrm{e}}$ & $\begin{array}{l}-1.21\left(38.5 \mathrm{~cm}^{-1}\right)^{\mathrm{e}} \\
{\left[33.2 \mathrm{~cm}^{-1}\right]^{\mathrm{f}}}\end{array}$ \\
\hline
\end{tabular}

${ }^{\text {a }}$ Percentage value relative to the $\Delta \mathrm{E}_{\text {ele-nuc }} \cdot\left(=\left(\Delta \mathrm{E}^{\mathrm{BSSE}} / \Delta \mathrm{E}_{\text {ele-nuc }}\right) \mathrm{x} 100\right){ }^{\mathrm{b}}$ Experimental value from refs. ${ }^{63,64,65} ;{ }^{\mathrm{c}}$ Hartree-Fock $(\mathrm{HF})$ contribution to the MP2 interaction energy $\left(\Delta \mathrm{E}^{\mathrm{MP} 2}=\Delta \mathrm{E}^{\mathrm{HF}}+\Delta \mathrm{E}^{(2)}\right)$; ${ }^{\mathrm{d}}$ Experimental value evaluated using the Pseudo-diatomic (PD) model (See for example ${ }^{62}$ and

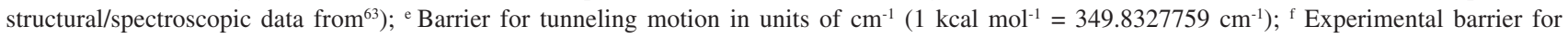
tunneling motion value from ref.. ${ }^{61} \mathrm{~V}_{4}$ barrier estimated from the splitting frequency of $2207 \mathrm{MHz}\left(0.0736 \mathrm{~cm}^{-1}\right)$ : $\mathrm{V}_{(\alpha)}=\mathrm{V}_{4} / 2[1+\cos (4 \alpha)] ;{ }^{\mathrm{g}} \mathrm{W}_{1}$ is the lowest harmonic frequency (in units of $\mathrm{cm}^{-1}$ ) which characterize the located stationary point as true minima or first-order TS structure.

when electron correlation is included at the MP4(SDTQ)/ $6-311++\mathrm{G}(3 \mathrm{df}, 3 \mathrm{pd})$ level of calculation. For the $(\mathrm{HCCH})_{2}$ dimer the PW91/6-311++G(3df,3pd) energy values agree well with the corresponding MP4(SDTQ) BSSE corrected results, indicating that the PW91 functional seems to encompass the correlation energy in this case.

The weakest van der Waals dimer investigated, the $\left(\mathrm{H}_{2}\right)_{2}$ complex, provided a good test for the ability of the DFT to describe electron correlation or dispersion effects. The results of the calculations are reported in Table 4 . In this case the PW91 functional does indeed overestimate considerably the stabilization energy compared to the best Post-HF calculations reported here and also the available experimental data, while, as could be expected, the BLYP functional does a very poor job to describe intermolecular interactions governed dominantly by dispersion effects 
Table 4. Geometrical parameters and interaction energy values for the $\left(\mathrm{H}_{2}\right)_{2}$ T-Shaped and Slipped-Parallel van der Waals dimers obtained with the standard Pople's split-valence basis sets. Distances in angstrom, angles in degrees and energies in units of kcal mol-1

\begin{tabular}{|c|c|c|c|c|c|c|}
\hline $\begin{array}{l}\left(\mathrm{H}_{2}\right)_{2} \\
\text { T-Shaped }\end{array}$ & $\begin{array}{l}\mathrm{HF} / 6-311++\mathrm{G} \\
(2 \mathrm{~d}, 2 \mathrm{p})\end{array}$ & $\begin{array}{l}\mathrm{HF} / 6-311++\mathrm{G} \\
\text { (3df,3pd) }\end{array}$ & $\begin{array}{l}\text { BLYP/6-311++G } \\
(2 \mathrm{~d}, 2 \mathrm{p})\end{array}$ & $\begin{array}{l}\text { BLYP/6-311++G } \\
\text { (3df,3pd) }\end{array}$ & $\begin{array}{l}\text { PW91/6-311++G } \\
(2 d, 2 p)\end{array}$ & $\begin{array}{l}\text { PW91/6-311++G } \\
\text { (3df,3pd) }\end{array}$ \\
\hline $\mathrm{R}_{\mathrm{CM}}$ & 4.230 & 4.188 & - & 7.385 & - & 3.274 \\
\hline$\theta_{\mathrm{A}}$ & 93.6 & 93.6 & - & 96.8 & - & 92.9 \\
\hline$\theta_{\mathrm{B}}$ & 175.7 & 175.7 & - & 170.2 & - & 177.6 \\
\hline$\phi$ & 180.0 & 180.0 & - & 180.0 & - & 180.0 \\
\hline $\mathrm{w}_{1}{ }^{\mathrm{e}}$ & 16.6 & 19.5 & - & $5.0 \mathrm{i}$ & - & 3.5 \\
\hline$\Delta \mathrm{E}_{\text {ele-nuc }}$ & -0.0112 & -0.0114 & - & -0.00075 & - & -0.30 \\
\hline$\Delta \mathrm{E}^{\text {ele-nSE }}$ & -0.0149 & -0.0170 & - & -0.00228 & - & -0.019 \\
\hline$\Delta \mathrm{E}^{\mathrm{BSSEc}}$ & - & - & - & - & - & - \\
\hline $\begin{array}{l}\left(\mathrm{H}_{2}\right)_{2} \\
\text { T-Shaped }\end{array}$ & $\begin{array}{l}\text { MP2/6-311++G } \\
(2 \mathrm{~d}, 2 \mathrm{p})\end{array}$ & $\begin{array}{l}\text { MP4(SDQ)/6-311++G } \\
(2 \mathrm{~d}, 2 \mathrm{p})\end{array}$ & $\begin{array}{l}\text { MP4(SDTQ)/6-311++G } \\
(2 \mathrm{~d}, 2 \mathrm{p})\end{array}$ & $\begin{array}{l}\text { MP2/6-311++G } \\
\text { (3df,3pd) }\end{array}$ & $\begin{array}{l}\text { MP4(SDQ)/6-311++G } \\
(3 \mathrm{df}, 3 \mathrm{pd})\end{array}$ & $\begin{array}{l}\text { MP4(SDTQ)/6-311++G } \\
(3 \mathrm{df}, 3 \mathrm{pd})\end{array}$ \\
\hline $\mathrm{R}_{\mathrm{CM}}$ & 3.574 & & & 3.469 & & \\
\hline$\theta_{\mathrm{A}}$ & 93.1 & & & 93.0 & & \\
\hline$\theta_{\mathrm{B}}^{\mathrm{A}}$ & 176.9 & & & 177.1 & & \\
\hline$\phi$ & 180.0 & & & 180.0 & & \\
\hline $\mathrm{w}_{1}{ }^{\mathrm{e}}$ & 31.4 & & & 33.2 & & \\
\hline$\Delta \mathrm{E}_{\text {ele-nuc }}$ & $\begin{array}{l}-0.05503 \\
\{0.01424\}\end{array}$ & -0.06055 & -0.06445 & $\begin{array}{l}-0.07944 \\
\{0.03169\}\end{array}$ & $\begin{array}{l}-0.09086 \\
\{-0.08352\}^{c}\end{array}$ & $\begin{array}{l}-0.09808 \\
\{-0.09475\}^{d}\end{array}$ \\
\hline$\Delta E^{\mathrm{BSSE}}$ & $\begin{array}{l}0.009977 \\
(18 \%)^{\mathrm{a}} \\
\{0.004330\}^{\mathrm{b}} \\
\{30 \%\}^{\mathrm{b}}\end{array}$ & $\begin{array}{l}0.01186 \\
(20 \%)^{\mathrm{a}}\end{array}$ & $\begin{array}{l}0.01186 \\
(18 \%)^{\mathrm{a}}\end{array}$ & $\begin{array}{l}0.01337 \\
(17 \%)^{\mathrm{a}} \\
\{0.003138\}^{\mathrm{b}} \\
\{10 \%\}^{\mathrm{b}}\end{array}$ & $\begin{array}{l}0.01544 \\
(17 \%)^{a} \\
\{0.01581\}^{c} \\
\{19 \%\}^{c}\end{array}$ & $\begin{array}{l}0.01544 \\
(16 \%)^{\mathrm{a}} \\
\{0.01581\}^{\mathrm{d}} \\
\{17 \%\}^{\mathrm{d}}\end{array}$ \\
\hline$\Delta \mathrm{E}^{\mathrm{BSSEc}}$ & $\begin{array}{l}-0.04505 \\
\{0.01857\}\end{array}$ & -0.04869 & -0.05259 & $\begin{array}{l}-0.06607 \\
\{0.03483\}\end{array}$ & $\begin{array}{l}-0.07542 \\
\{-0.06771\}^{c}\end{array}$ & $\begin{array}{l}-0.08264 \\
\{-0.07894\}\end{array}$ \\
\hline $\begin{array}{l}\left(\mathrm{H}_{2}\right)_{2} \\
\text { Slipp-Par }\end{array}$ & $\begin{array}{l}\mathrm{HF} / 6-311++\mathrm{G} \\
(2 \mathrm{~d}, 2 \mathrm{p})\end{array}$ & $\begin{array}{l}\mathrm{HF} / 6-311++\mathrm{G} \\
(3 \mathrm{df}, 3 \mathrm{pd})\end{array}$ & $\begin{array}{l}\text { BLYP/6-311++G } \\
(2 \mathrm{~d}, 2 \mathrm{p})\end{array}$ & $\begin{array}{l}\text { BLYP/6-311++G } \\
\text { (3df,3pd) }\end{array}$ & $\begin{array}{l}\text { PW91/6-311++G } \\
(2 \mathrm{~d}, 2 \mathrm{p})\end{array}$ & $\begin{array}{l}\text { PW91/6-311++G } \\
\text { (3df,3pd) }\end{array}$ \\
\hline $\mathrm{R}_{\mathrm{CM}}$ & 4.270 & 4.252 & - & 6.682 & - & 3.247 \\
\hline$\theta_{\mathrm{A}}$ & 50.1 & 50.1 & - & 51.7 & - & 48.9 \\
\hline$\theta_{\mathrm{B}}$ & 130.9 & 130.8 & - & 133.6 & - & 130.5 \\
\hline$\phi$ & 0.0 & 0.0 & - & 0.0 & - & 0.0 \\
\hline $\mathrm{w}_{1}^{\mathrm{e}}$ & $16.8 \mathrm{i}$ & $18.0 \mathrm{i}$ & - & $8.2 \mathrm{i}$ & - & 26.8 \\
\hline$\Delta \mathrm{E}_{\text {ele-nuc }}$ & -0.00935 & -0.00922 & - & -0.00050 & - & -0.29 \\
\hline$\Delta \mathrm{E}^{\mathrm{BSSE}}$ & -0.0147 & -0.0173 & - & -0.0226 & - & -0.018 \\
\hline$\Delta \mathrm{E}^{\mathrm{BSSEc}}$ & - & - & - & - & - & - \\
\hline $\begin{array}{l}\left(\mathrm{H}_{2}\right)_{2} \\
\text { Slipp-Par }\end{array}$ & $\begin{array}{l}\text { MP2/6-311++G } \\
(2 \mathrm{~d}, 2 \mathrm{p})\end{array}$ & $\begin{array}{l}\text { MP4(SDQ)/6-311++G } \\
(2 \mathrm{~d}, 2 \mathrm{p})\end{array}$ & $\begin{array}{l}\text { MP4(SDTQ)/6-311++G } \\
(2 \mathrm{~d}, 2 \mathrm{p})\end{array}$ & $\begin{array}{l}\text { MP2/6-311++G } \\
(3 \mathrm{df}, 3 \mathrm{pd})\end{array}$ & $\begin{array}{l}\text { MP4(SDQ)/6-311++G } \\
(3 \mathrm{df}, 3 \mathrm{pd})\end{array}$ & $\begin{array}{l}\text { MP4(SDTQ)/6-311++G } \\
(3 \mathrm{df}, 3 \mathrm{pd})\end{array}$ \\
\hline $\mathrm{R}_{\mathrm{CM}}$ & 3.684 & & & 3.491 & & \\
\hline$\theta_{\mathrm{A}}$ & 44.3 & & & 49.5 & & \\
\hline$\theta_{\mathrm{B}}$ & 135.0 & & & 130.1 & & \\
\hline$\phi$ & 0.0 & & & 0.0 & & \\
\hline $\mathrm{w}_{1}{ }^{\mathrm{e}}$ & $29.5 \mathrm{i}$ & & & $24.7 \mathrm{i}$ & & \\
\hline$\Delta \mathrm{E}_{\text {ele-nuc }}$ & $\begin{array}{l}-0.04882 \\
\{0.007844\}\end{array}$ & -0.05359 & -0.05679 & $\begin{array}{l}-0.07103 \\
\{0.03043\}\end{array}$ & $\begin{array}{l}-0.08233 \\
\{-0.07549\}^{c}\end{array}$ & $\begin{array}{l}-0.08879 \\
\{-0.08559\} d\end{array}$ \\
\hline$\Delta E^{\mathrm{BSSE}}$ & $\begin{array}{l}0.008660 \\
(18 \%)^{\mathrm{a}} \\
\{0.004518\}^{\mathrm{b}} \\
\{58 \%\}^{\mathrm{b}}\end{array}$ & $\begin{array}{l}0.01042 \\
(19 \%)^{\mathrm{a}}\end{array}$ & $\begin{array}{l}0.01042 \\
(18 \%)^{\mathrm{a}}\end{array}$ & $\begin{array}{l}0.01261 \\
(18 \%)^{\mathrm{a}} \\
\{0.003200\}^{\mathrm{b}} \\
\{11 \%\}^{\mathrm{b}}\end{array}$ & $\begin{array}{l}0.01475 \\
(18 \%)^{\mathrm{a}} \\
\{0.01481\}^{\mathrm{c}} \\
\{20 \%\}^{\mathrm{c}}\end{array}$ & $\begin{array}{l}0.01475 \\
(17 \%)^{\mathrm{a}} \\
\{0.01481\}^{\mathrm{d}} \\
\{17 \%\}^{\mathrm{d}}\end{array}$ \\
\hline$\Delta \mathrm{E}^{\mathrm{BSSEc}}$ & $\begin{array}{l}-0.04016 \\
\{0.01236\}\end{array}$ & -0.04317 & -0.04637 & $\begin{array}{l}-0.05842 \\
\{0.03363\}\end{array}$ & $\begin{array}{l}-0.06758 \\
\{-0.06068\}^{c}\end{array}$ & $\begin{array}{l}-0.07404 \\
\{-0.07078\}\end{array}$ \\
\hline
\end{tabular}

${ }^{\text {a }}$ Percentage value relative to the $\Delta \mathrm{E}_{\text {ele-nuc }} \cdot\left(=\left(\Delta \mathrm{E}^{\mathrm{BSSE}} / \Delta \mathrm{E}_{\text {ele-nuc }}\right) \mathrm{x} 100\right)$; ${ }^{\mathrm{b}}$ Hartree-Fock $(\mathrm{HF})$ contribution to the MP2 interaction energy $\left(\Delta \mathrm{E}^{\mathrm{MP} 2}=\Delta \mathrm{E}^{\mathrm{HF}}\right.$ $\left.+\Delta \mathrm{E}^{(2)}\right) ;{ }^{\mathrm{c}} \mathrm{CCSD}$ value; ${ }^{\mathrm{d}} \mathrm{CCSD}-\mathrm{T}$ value; ${ }^{\mathrm{e}} \mathrm{W}_{1}$ is the lowest harmonic frequency (in units of $\mathrm{cm}^{-1}$ ) which characterize the located stationary point as true minima or first-order TS structure.

(similar to the behavior of the HF method). It can be seen from Table 4 that the BSSE is still sizeable for the 6$311++\mathrm{G}(3 \mathrm{df}, 3 \mathrm{pd})$ basis set, with the aug-cc-pVTZ basis set doing a similar job (see Table 5). However, raising the basis set quality to the quintuple-zeta, i.e., aug-cc-pV5Z, the BSSE correction is below $1 \%$ at the MP4(SDTQ) level, which gives a strong support to the use of this basis set to investigate weakly bound van der Waals dimers, at a higher correlated level of theory. It may be inferred that this BSSE pattern is an indication of the balanced behavior of the basis set, providing a criterion to assess the quality of a given basis set to be used in molecular interaction studies. 
Table 5. Geometrical parameters and interaction energy values for the $\left(\mathrm{H}_{2}\right)_{2}$ T-Shaped and Slipped-Parallel van der Waals dimers obtained with the Dunning's correlated consistent polarized valence basis sets. Distances in angstrom, angles in degrees and energies in units of kcal mol ${ }^{-1}$

\begin{tabular}{|c|c|c|c|c|c|c|}
\hline $\begin{array}{l}\left(\mathrm{H}_{2}\right)_{2} \\
\text { T-Shaped }\end{array}$ & $\begin{array}{l}\text { MP2/ } \\
\text { aug-cc-pVTZ }\end{array}$ & $\begin{array}{l}\text { MP4(SDQ)/ } \\
\text { aug-cc-pVTZ }\end{array}$ & $\begin{array}{l}\text { MP4(SDTQ)/ } \\
\text { aug-cc-pVTZ }\end{array}$ & $\begin{array}{l}\text { MP2/ } \\
\text { aug-cc-pVQZ }\end{array}$ & $\begin{array}{l}\text { MP4(SDQ)/ } \\
\text { aug-cc-pVQZ }\end{array}$ & $\begin{array}{l}\text { MP4(SDTQ)/ } \\
\text { aug-cc-pVQZ }\end{array}$ \\
\hline $\mathrm{R}_{\mathrm{CM}}$ & 3.404 & & & 3.406 & & \\
\hline$\theta_{\mathrm{A}}$ & 93.0 & & & 92.9 & & \\
\hline$\theta_{\mathrm{B}}^{\mathrm{A}}$ & 177.3 & & & 177.2 & & \\
\hline$\phi$ & 180.0 & & & 180.0 & & \\
\hline $\mathrm{w}_{1}{ }^{\mathrm{i}}$ & 59.4 & & & 37.9 & & \\
\hline$\Delta \mathrm{E}_{\text {ele-nuc }}$ & $\begin{array}{l}-0.0973 \\
\{0.04091\}^{b}\end{array}$ & $\begin{array}{l}0.1070 \\
-\{-0.09808\}^{c}\end{array}$ & $\begin{array}{l}-0.1165 \\
\{-0.1126\}^{d}\end{array}$ & $\begin{array}{l}-0.0892 \\
\{0.04510\}\end{array}$ & $\begin{array}{l}-0.09745 \\
\{-0.08804\}^{c}\end{array}$ & $\begin{array}{l}-0.1076 \\
\{-0.1034\}^{d}\end{array}$ \\
\hline \multirow[t]{4}{*}{$\Delta \mathrm{E}^{\mathrm{BSSE}}$} & 0.01989 & 0.01826 & 0.01826 & 0.006212 & 0.004142 & 0.004142 \\
\hline & $(20.4 \%)^{\mathrm{a}}$ & $(17.1 \%)^{\mathrm{a}}$ & $(15.7 \%)^{\mathrm{a}}$ & $(7.0 \%)^{\mathrm{a}}$ & $(4.2 \%)^{\mathrm{a}}$ & $(3.8 \%)^{\mathrm{a}}$ \\
\hline & $\{0.007969\}^{b}$ & $\{0.01876\}^{\mathrm{c}}$ & $\{0.01876\}^{\mathrm{d}}$ & $\{0.001632\}\}^{b}$ & $\{0.004142\}^{\mathrm{c}}$ & $\{0.004142\}^{d}$ \\
\hline & $\{19 \%\}^{b}$ & $\{19.1 \%\}^{\mathrm{c}}$ & $\{16.7 \%\}^{\mathrm{d}}$ & $\{4 \%\}^{b}$ & $\{4.7 \%\}^{\mathrm{c}}$ & $\{4.0 \%\}^{\mathrm{d}}$ \\
\hline$\Delta \mathrm{E}^{\mathrm{BSSEc}}$ & $-0.07741\{0.04888\}^{b}$ & $-0.08874\{-0.07932\}^{\mathrm{c}}$ & $-0.09824\{-0.09384\}^{\mathrm{d}}$ & $-0.08299\{0.04673\}^{b}$ & $-0.09331\{-0.08390\}^{c}$ & $-0.1035\{-0.09926\}^{d}$ \\
\hline $\begin{array}{l}\left(\mathrm{H}_{2}\right)_{2} \\
\text { Slipp-Par }\end{array}$ & $\begin{array}{l}\text { MP2/ } \\
\text { aug-cc-pVTZ }\end{array}$ & $\begin{array}{l}\text { MP4(SDQ)/ } \\
\text { aug-cc-pVTZ }\end{array}$ & $\begin{array}{l}\text { MP4(SDTQ)/ } \\
\text { aug-cc-pVTZ }\end{array}$ & $\begin{array}{l}\text { MP2/ } \\
\text { aug-cc-pVQZ }\end{array}$ & $\begin{array}{l}\text { MP4(SDQ)/ } \\
\text { aug-cc-pVQZ }\end{array}$ & $\begin{array}{l}\text { MP4(SDTQ)/ } \\
\text { aug-cc-pVQZ }\end{array}$ \\
\hline$R_{\mathrm{CM}}$ & 3.471 & & & 3.425 & & \\
\hline$\theta_{\mathrm{A}}$ & 49.68 & & & 49.5 & & \\
\hline$\theta_{\mathrm{B}}^{\mathrm{A}}$ & 130.0 & & & 130.0 & & \\
\hline$\phi$ & 0.0 & & & 0.0 & & \\
\hline $\mathrm{w}_{1}{ }^{\mathrm{i}}$ & $30.5 \mathrm{i}$ & & & $35.4 \mathrm{i}$ & & \\
\hline$\Delta \mathrm{E}_{\text {ele-nuc }}$ & $\begin{array}{l}-0.08540 \\
\{0.03150\}\end{array}$ & $\begin{array}{l}-0.09425 \\
\{-0.08678\}^{c}\end{array}$ & $\begin{array}{l}-0.1022 \\
\{-0.09902\} d\end{array}$ & $\begin{array}{l}-0.08020 \\
\{0.04499\}\end{array}$ & $\begin{array}{l}-0.08886 \\
\{-0.08020\}^{c}\end{array}$ & $\begin{array}{l}-0.09814 \\
\{-0.09425\} d\end{array}$ \\
\hline \multirow[t]{4}{*}{$\Delta \mathrm{E}^{\mathrm{BSSE}}$} & 0.01581 & 0.01412 & 0.01412 & 0.005522 & 0.003640 & 0.003640 \\
\hline & $(19 \%)^{\mathrm{a}}$ & $(15 \%)^{\mathrm{a}}$ & $(14 \%)^{\mathrm{a}}$ & $(7 \%)^{\mathrm{a}}$ & $(4 \%)^{\mathrm{a}}$ & $(4 \%)^{\mathrm{a}}$ \\
\hline & $\{0.006024\}^{b}$ & $\{0.01456\}^{\mathrm{c}}$ & $\{0.01456\}^{d}$ & $\{0.001757\}^{b}$ & $\{0.003640\}^{\mathrm{c}}$ & $\{0.003640\}^{\mathrm{d}}$ \\
\hline & $\{19 \%\}^{b}$ & $\{17 \%\}^{\mathrm{c}}$ & $\{14.7 \%\}^{\mathrm{d}}$ & $\{4 \%\}^{\mathrm{b}}$ & $\{4.5 \%\}^{\mathrm{c}}$ & $\{3.9 \%\}^{\mathrm{d}}$ \\
\hline \multirow[t]{4}{*}{$\Delta \mathrm{E}^{\mathrm{BSSEc}}$} & -0.06959 & -0.08013 & -0.08808 & -0.07468 & -0.08522 & -0.0945 \\
\hline & $\{0.03752\}^{\mathrm{b}}$ & $\{-0.07222\}^{c}$ & $\{-0.08446\}^{d}$ & $\{0.04675\}^{\mathrm{b}}$ & $\{-0.07656\}^{\mathrm{c}}$ & $\{-0.09061\}^{d}$ \\
\hline & $\left(\mathrm{H}_{2}\right)_{2}$ T-Shaped & & & $\left(\mathrm{H}_{2}\right)_{2}$ Slipped-Parallel & & \\
\hline & $\begin{array}{l}\text { MP2/aug-cc-pV5Z } \\
\text { //MP2/aug-cc-pVQZ }\end{array}$ & $\begin{array}{l}\text { MP4(SDQ)/ } \\
\text { aug-cc-pV5Z } \\
\text { //MP2/aug-cc-pVQZ }\end{array}$ & $\begin{array}{l}\text { MP4(SDTQ)/ } \\
\text { aug-cc-pV5Z } \\
\text { //MP2/aug-cc-pVQZ }\end{array}$ & $\begin{array}{l}\text { MP2/aug-cc-pV5Z } \\
\text { //MP2/aug-cc-pVQZ }\end{array}$ & $\begin{array}{l}\text { MP4(SDQ)/ } \\
\text { aug-cc-pV5Z } \\
\text { //MP2/aug-cc-pVQZ }\end{array}$ & $\begin{array}{l}\text { MP4(SDTQ)/ } \\
\text { aug-cc-pV5Z } \\
\text { //MP2/aug-cc-pVQZ }\end{array}$ \\
\hline$\overline{\Delta \mathrm{E}_{\text {ele-nuc }}}$ & $\begin{array}{l}-0.08716 \\
\{0.04757\}\end{array}$ & $\begin{array}{l}-0.09532 \\
\{-0.08597\}^{c}\end{array}$ & $\begin{array}{l}-0.1057 \\
\{-0.1016\} d\end{array}$ & $\begin{array}{l}-0.07850 \\
\{0.04662\}\end{array}$ & $\begin{array}{l}-0.08722 \\
\{-0.07863\}^{c}\end{array}$ & $\begin{array}{l}-0.09670 \\
\{-0.09293\} \text { d }\end{array}$ \\
\hline \multirow[t]{4}{*}{$\Delta \mathrm{E}^{\mathrm{BSSE}}$} & 0.002573 & 0.001004 & 0.001004 & 0.002008 & 0.000753 & 0.000753 \\
\hline & $(3 \%)^{\mathrm{a}}$ & $(1 \%)^{\mathrm{a}}$ & $(0.9 \%)^{\mathrm{a}}$ & $(3 \%)^{\mathrm{a}}$ & $(0.9 \%)^{\mathrm{a}}$ & $(0.8 \%)^{\mathrm{a}}$ \\
\hline & $\{0.0001255\}^{\mathrm{b}}$ & $\{0.001067\}^{\mathrm{c}}$ & $\{0.001067\}^{\mathrm{d}}$ & $\{0.0001255\}^{b}$ & $\{0.000753\}^{\mathrm{c}}$ & $\{0.000753\}^{\mathrm{d}}$ \\
\hline & $\{0.3 \%\}^{\mathrm{b}}$ & $\{1.2 \%\}^{\mathrm{c}}$ & $\{1.0 \%\}^{\mathrm{d}}$ & $\{0.3 \%\}^{b}$ & $\{1.0 \%\}^{c}$ & $\{0.8 \%\}^{\mathrm{d}}$ \\
\hline \multirow[t]{6}{*}{$\Delta \mathrm{E}^{\mathrm{BSSEc}}$} & -0.0846 & -0.0943 & -0.1047 & -0.07649 & -0.08647 & -0.0959 \\
\hline & $\{0.04770\}^{b}$ & $\{-0.08490\}^{c}$ & $\{-0.1005\}^{d}$ & $\{0.04675\}^{\mathrm{b}}$ & $\{-0.07788\}^{c}$ & $\{-0.09218\}^{d}$ \\
\hline & & & {$[-0.0717]^{\mathrm{e}}$} & & & \\
\hline & & & {$[-0.0735]^{\mathrm{f}}$} & & & \\
\hline & & & {$[-0.0741]^{g}$} & & & \\
\hline & & & {$[-0.0692]^{\mathrm{h}}$} & & & \\
\hline
\end{tabular}

${ }^{\text {a }}$ Percentage value relative to the $\Delta \mathrm{E}_{\text {ele-nuc }} \cdot\left(=\left(\Delta \mathrm{E}^{\mathrm{BSSE}} / \Delta \mathrm{E}_{\text {ele-nuc }}\right) \mathrm{x} 100\right) ;{ }^{\mathrm{b}}$ Hartree-Fock $(\mathrm{HF})$ contribution to the $\mathrm{MP} 2$ interaction energy $\left(\Delta \mathrm{E}^{\mathrm{MP} 2}=\Delta \mathrm{E}^{\mathrm{HF}}\right.$ $\left.+\Delta \mathrm{E}^{(2)}\right)$; ${ }^{\mathrm{c}} \mathrm{CCSD}$ value; ${ }^{\mathrm{d}} \mathrm{CCSD}-\mathrm{T}$ value; ${ }^{\circ}$ Experimental value from refs. ${ }^{66,67}$; f,g Experimental value obtained from the transport properties; ${ }^{68,69}$ ${ }^{\mathrm{h}}$ Experimental value obtained from molecular beams experiment. ${ }^{70} ;{ }^{\mathrm{i}} \mathrm{w}_{1}$ is the lowest harmonic frequency (in units of $\mathrm{cm}^{-1}$ ) which characterize the located stationary point as true minima or TS structure.

It should be noted that our best ab initio BSSE corrected stabilization energy value of $-0.105 \mathrm{kcal} \mathrm{mol}^{-1}$ (MP4(SDTQ)/aug-cc-pV5Z//MP2/aug-cc-pVQZ) still deviates considerably from the available experimental data of $-0.072 \mathrm{kcal} \mathrm{mol}^{-1}$. However, the experimental uncertainty and limitation must be considered, once we are surely on the basis set limit and the electron correlation is adequately included. Also the stabilization energy value is quite small $\left(<1 \mathrm{kcal} \mathrm{mol}^{-1}\right)$ which certainly offer a great challenge for its accurate experimental determination, being also very sensitive to the experimental method utilized for its determination as can be seen from the range of values quoted in Table 5.

In order the compare the performance of Post-HF methods to describe electron correlation effects CCSD-T single point energy calculations were performed for the MP2 optimized geometries using the Pople's split-valence and Dunning's correlated consistent polarized valence basis sets for the $\left(\mathrm{H}_{2}\right)_{2}$ dimer, which is the smallest dimer investigated here, so our computational resources enable 
such huge $a b$ initio calculation to be carried out. The conclusions can be extended to other weakly bound dimers. With all basis sets utilized the CCSD-T stabilization energies are systematically smaller than the MP4(SDTQ) ones by $c a$. 3-4\% (0.004 kcal mol-1). The comparison between the CCSD and MP4(SDQ) energies shows a larger variation of $c a$. 9-11\%, with the CCSD being also smaller. In addition, the BSSE correction is virtually the same, so what would differs the two correlated calculations is indeed the way the electron correlation is handled, since both treatment of the correlation effects exhibit the same dependence with the basis set. It can be seen that no matter how big is the basis set this regular patter is observed for both energies and BSSE correction. So, it the light of these results it can be said that the CCSD$\mathrm{T}$ and MP4(SDTQ) stabilization energies agree within 3$4 \%$, which is probably closer to the lowest experimental uncertainty for interaction energy determination for weakly bound dimers. In order to establish definitively whether or not the MP4(SDTQ) interaction energies are overestimated a systematic study for a series of weakly bound dimers, where experimental energies with uncertainties included are available, would be required. As the MP4(SDTQ) approach is computationally much cheaper than the CCSD-T one, the results reported in this article stimulates the use of the MP4 treatment for electron correlation in theoretical studies of weak molecular interactions in the gas phase.

In order to have a further assessment of the dispersion contribution to the interaction energies the multipole expansion described previously was used for the description of the long-range forces which play an important role for weakly bound species. One dimensional potential curves were also constructed by calculating the $a b$ initio energies as a function of the center of mass intermolecular distance, varying the distance from 2 to 9 angstrom, hence including part of the short-range region. In these calculations the MP2/6-311++G(2d,2p) level was used, with all geometrical parameters being fully optimized without any constraint, and the center of mass distance kept frozen in the range of 2-9 angstrom, with a stepsize of 0.5 angstrom. The results are shown in Figures 3-5, respectively for the $\left(\mathrm{H}_{2}\right)_{2}$-T-Shaped, $\mathrm{HCN}$-linear, $(\mathrm{HCCH})_{2}-$ T-Shaped and $\left(\mathrm{H}_{2} \mathrm{O}\right)_{2}$ dimers. In these plots the HartreeFock contribution to the MP2 energy curve is also shown, along with the Lennard-Jones (12-6) representation of the potential, ${ }^{71}$ using the MP2 parameters. The functional form of the LJ-(12-6) potential is given below, wher $-\varepsilon$ is the minimum energy value at the equilibrium distance $\mathrm{r}_{\mathrm{eq}}$ and $\sigma\left(=2^{-1 / 6} r_{\text {eq }}\right)$ is the intermolecular separation for which the interaction energy is zero.
$U(r)=4 \varepsilon\left[\left(\frac{\sigma}{r}\right)^{12}-\left(\frac{\sigma}{r}\right)^{6}\right]$

The HF contribution to the Post-HF stabilization energies are also given in Tables 1-5, along with the corresponding HF energies for the fully optimized HF equilibrium structures. The size of the HF and MPn contributions can be evaluated at the minimum energy point and the way these contributions vary on going from the short- to the long-range region can be seen from the potential curves depicted in Figures 2-5. As could be anticipated the weaker is the molecular complex the more important is the electron correlation effects. For the $\left(\mathrm{H}_{2}\right)_{2}$ dimer the very small size of the stabilization energy preclude the visualization of the electron correlation effects from Figure $3 \mathrm{a}$ (the scale has to be enlarged).
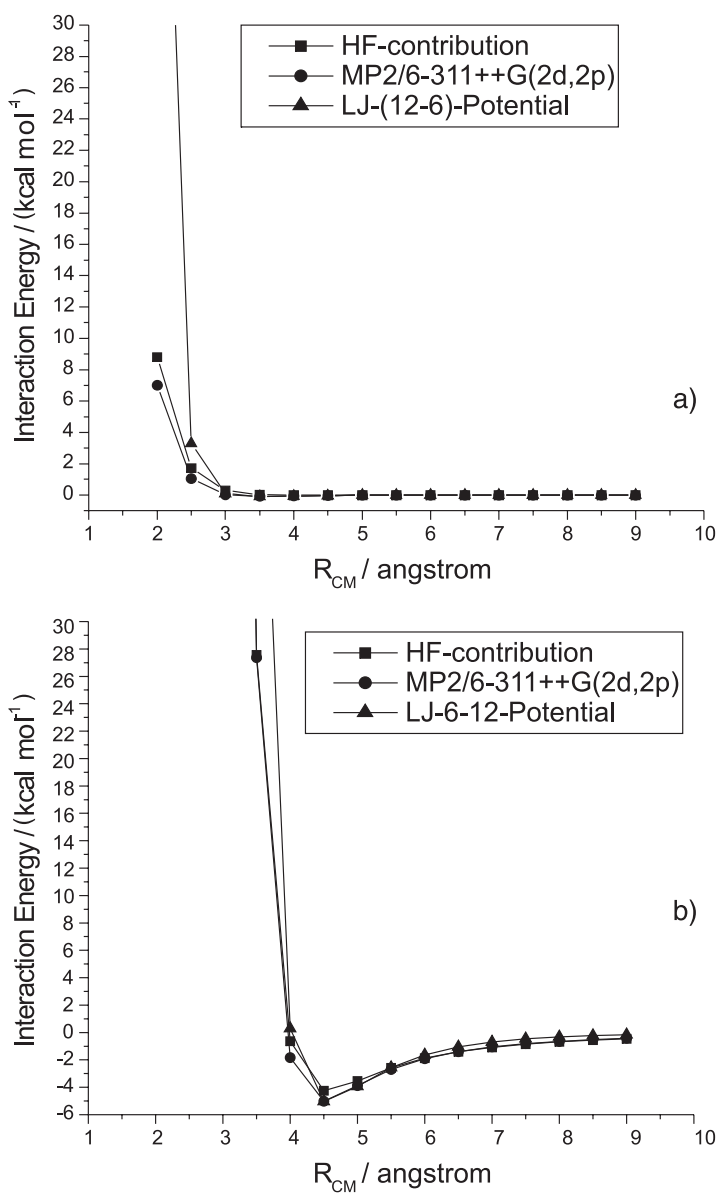

Figure 3. MP2/6-311++G(2d,2p) Potential Energy Curve. $R_{C M}$ is the center of mass distance between the two monomer. All geometrical parameters, except for the $\mathrm{R}_{\mathrm{CM}}$ distance, were fully optimized for each point on the energy curve. The Lennard-Jones (LJ) 12-6 Potential was plotted using the MP2/6-311++G(2d,2p) parameters. There were no significant angular distortions from the symmetric TShaped structure of the $\left(\mathrm{H}_{2}\right)_{2}$ dimer and Linear structure of the $(\mathrm{HCN})_{2}$ dimers, in the short distance region. (a) $\left(\mathrm{H}_{2}\right)_{2}$ T-Shaped dimer; (b) $(\mathrm{HCN})_{2}$ Linear dimer 

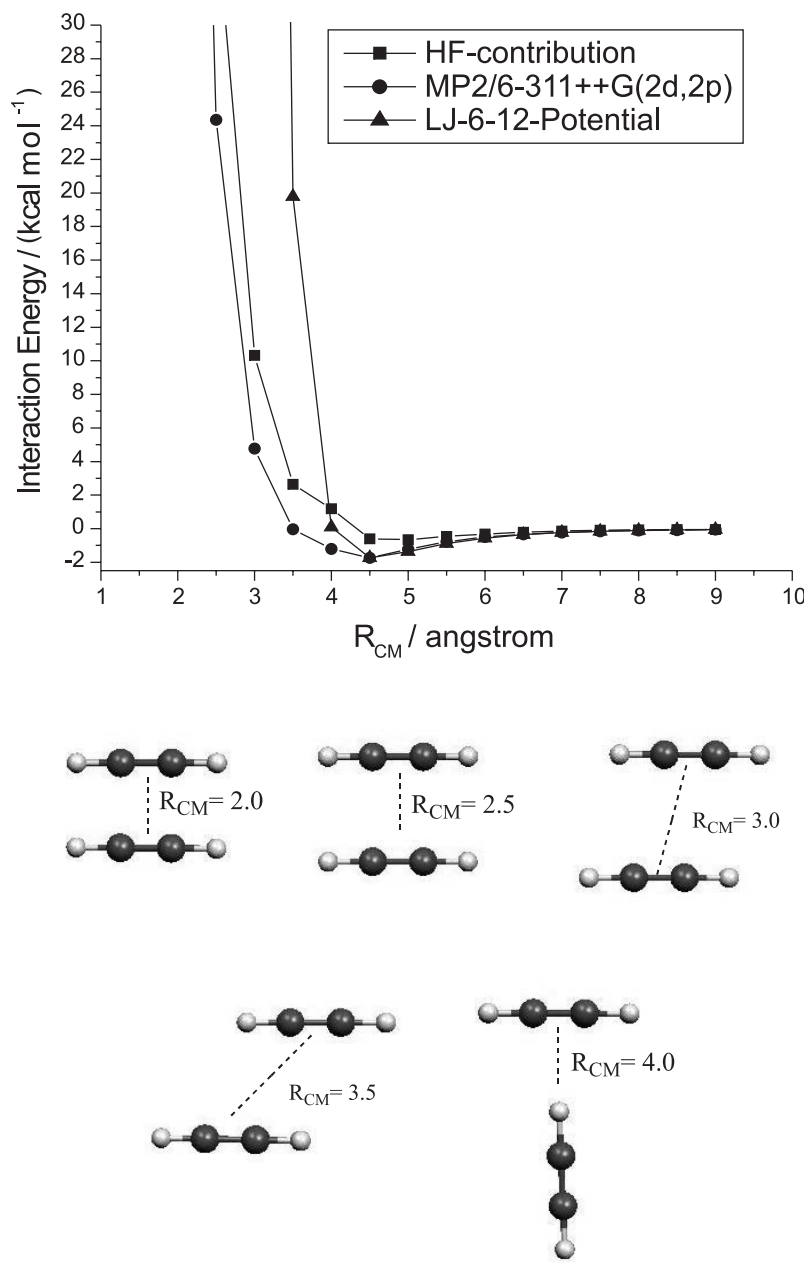

Figure 4: MP2/6-311++G(2d,2p) Potential Energy Curve for $(\mathrm{HCCH})$, T-Shaped dimer (as described in Figure 3 ). The preferred spatial orientation in the short distance region is shown.

It can be seen from Figure 4 that the electron correlation effects play an important role at the equilibrium and shortrange region, while at longer intermolecular distances the HF and MP2 are practically coincident. It is also shown that below 4 angstrom the T-Shaped structure of the $(\mathrm{HCCH})_{2}$ dimer is converted into the Slipped-Parallel one and then turning into the symmetrical Parallel structure. Therefore, any potential function to be used to model the behavior in the short-range region should be able to describe the angular dependence apart from the radial part as given by the Lennard-Jones potential. The same situation holds for the water dimer potential curve shown in Figure 5. Significant deviation from the $\mathrm{C}_{2 \mathrm{v}}$-Trans-linear structure occurs below 3 angstrom. Analyzing these potential energy curves it can be said that the LJ-(12-6) radial potential describes very well the intermolecular potential at the equilibrium and long-range region. However, a large deviation occur in the short-range region particularly for the $\left(\mathrm{H}_{2}\right)_{2}$ and $(\mathrm{HCCH})_{2}$ weakly bound dimers.
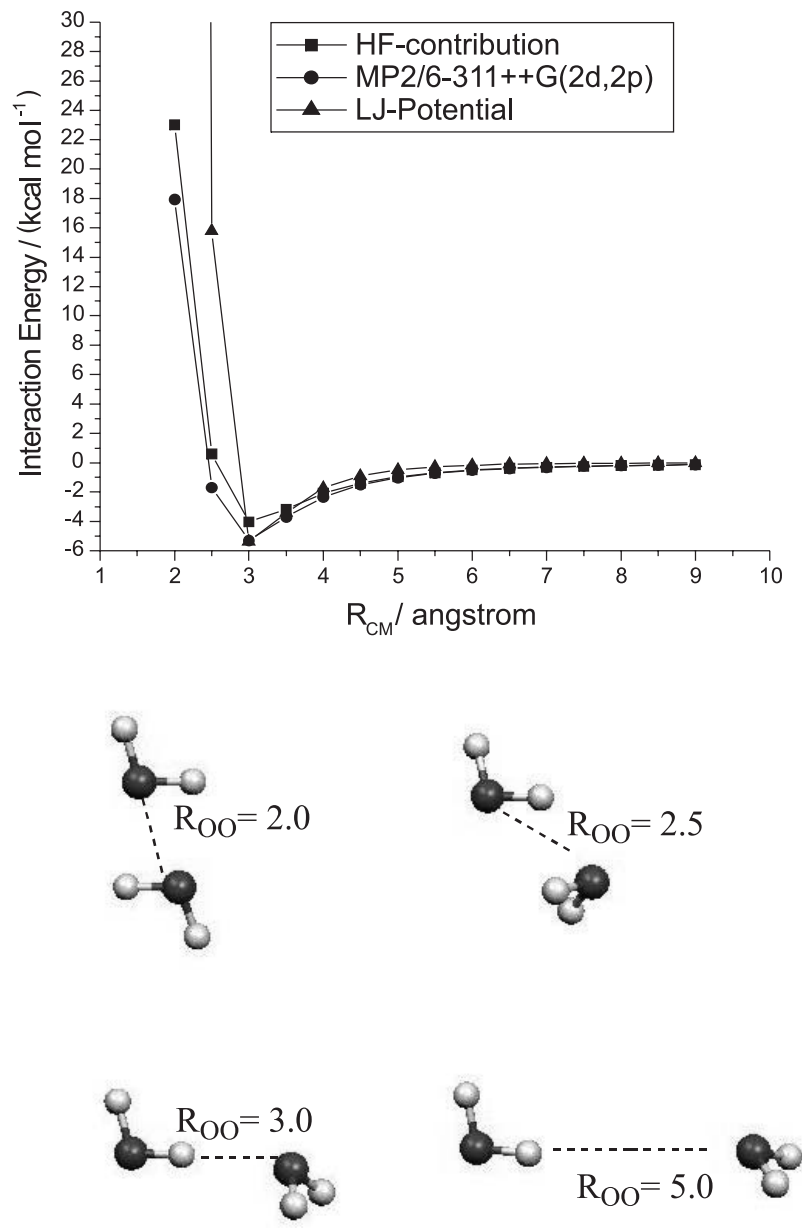

Figure 5: $\mathrm{MP} 2 / 6-311++\mathrm{G}(2 \mathrm{~d}, 2 \mathrm{p})$ Potential Energy Curve for $\left(\mathrm{H}_{2} \mathrm{O}\right)_{2}$ dimer (as described in Figure 3 ). The preferred spatial orientation in the short distance region is shown.

The electrostatic, induction and dispersion multipole expansion energy contributions to the long-range interaction energy are reported in Table 6. The electrical properties for the isolated monomers subunits and the dispersion coefficients are given respectively in Tables 7 and 8 . From the values for $-\mathrm{C}_{6}$ asym $/ \mathrm{R}^{6}$ reported in Table 6 (in parenthesis), which takes into account a deviation from the isotropicity of the polarizability tensor (equations (19) and (20)), it can be concluded that for T-Shaped and Slipped-Parallel spatial arrangements the simple London approximation ${ }^{47}$ works fine. However for the water dimer and linear $(\mathrm{HCN})_{2}$ structures the asymmetry characteristic given by $\mathrm{C}_{6}{ }^{\text {asym }}$ becomes relevant. This can be better observed by looking at the dispersion coefficients given in Table 8, where it can be seen that $\mathrm{C}_{6}$ asym does not change appreciably for T-Shaped and Slipped-Parallel spatial arrangements, but for water dimer it is almost half of the $\mathrm{C}_{6}$ value. Nevertheless, the neat effect on the total interaction energy $\left(\mathrm{U}_{\text {Total }}\right)$ is not so pronounced. The higher dispersion 
Table 6. Coulombic, Induction and Dispersion Long-Range Contributions to the Interaction Energy. All energy values in units of kcal mol ${ }^{-1}$. All $a b$ initio geometries were calculated at the MP2/6-311++G(2d,2p) level of theory

\begin{tabular}{|c|c|c|c|c|c|c|c|c|c|}
\hline $\begin{array}{l}\left(\mathrm{H}_{2} \mathrm{O}\right)_{2} \\
\text { Trans-Linear }\end{array}$ & $\begin{array}{l}\mathrm{U}_{\text {dipole }} \\
\mathrm{C}_{3} / \mathrm{R}^{3}\end{array}$ & $\begin{array}{l}\mathrm{U}_{\text {dip-quad }} \\
\mathrm{C}_{4} / \mathrm{R}^{4}\end{array}$ & $\begin{array}{l}\mathrm{U}_{\text {quadrupole }} \\
\mathrm{C}_{5} / \mathrm{R}^{5}\end{array}$ & $\begin{array}{l}\mathrm{U}_{\text {induction }} \\
-\mathrm{C}_{6}^{\text {ind }} / \mathrm{R}^{6}\end{array}$ & $\begin{array}{l}\mathrm{U}_{\text {dispersion }} \\
-\mathrm{C}_{6} / \mathrm{R}^{6}\end{array}$ & $\begin{array}{l}\mathrm{U}_{\text {disp-CB }} \\
-\mathrm{C}_{8} / \mathrm{R}^{8}\end{array}$ & $\begin{array}{l}\mathrm{U}_{\text {disp-C10 }} \\
-\mathrm{C}_{10} / \mathrm{R}^{10}\end{array}$ & $\mathrm{U}_{\text {Total }}$ & $\begin{array}{l}\text { MP4SDTQ/ } \\
6-311++G \text { } \\
(3 \mathrm{df}, 3 \mathrm{pd}){ }^{\mathrm{b}}\end{array}$ \\
\hline $\mathbf{A}^{\mathrm{d}}$ & -2.47 & -0.022 & -0.0024 & -0.21 & -0.68 & -0.50 & -0.19 & -4.07 & \\
\hline $\mathbf{B}^{\mathrm{e}}$ & -2.74 & 0.0014 & -0.0016 & -0.23 & $\begin{array}{l}-0.78 \\
(-0.36)^{a}\end{array}$ & -0.63 & -0.26 & -4.64 & $\begin{array}{l}-4.59 \\
{[-4.96]^{c}}\end{array}$ \\
\hline $\begin{array}{l}(\mathrm{HCN})_{2} \\
\text { Linear }\end{array}$ & $\begin{array}{l}\mathrm{U}_{\text {dipole }} \\
\mathrm{C}_{3} / \mathrm{R}^{3}\end{array}$ & $\begin{array}{l}\mathrm{U}_{\text {dip-quad }} \\
\mathrm{C}_{4} / \mathrm{R}^{4}\end{array}$ & $\begin{array}{l}\mathrm{U}_{\text {quadrupole }} \\
\mathrm{C}_{5} / \mathrm{R}^{5}\end{array}$ & $\begin{array}{l}\mathrm{U}_{\text {induction }} \\
-\mathrm{C}_{6} \text { ind } / \mathrm{R}^{6}\end{array}$ & $\begin{array}{l}U_{\text {dispersion }} \\
-C_{6} / R^{6}\end{array}$ & $\begin{array}{l}U_{\text {disp-C8 }} \\
-C_{8} / R^{8}\end{array}$ & $\begin{array}{l}U_{\text {disp-C10 }} \\
-C_{10} / R^{10}\end{array}$ & $\mathrm{U}_{\text {Total }}$ & $\begin{array}{l}\text { MP4SDTQ/ } \\
6-311++\mathrm{G} \\
(3 \mathrm{df}, 3 \mathrm{pd})^{\mathrm{b}}\end{array}$ \\
\hline$A^{d}$ & -2.616 & 0.0 & 2.9637 & -0.1546 & $\begin{array}{l}-0.2085 \\
(-0.2034)^{\mathrm{a}}\end{array}$ & -0.1311 & -0.04330 & -0.190 & \\
\hline $\mathbf{B}^{\mathrm{e}}$ & -2.989 & 0.0 & 0.2465 & -0.1696 & $\begin{array}{l}-0.1879 \\
(-0.08781)^{\mathrm{a}}\end{array}$ & -0.1109 & -0.03438 & -3.163 & -4.48 \\
\hline $\begin{array}{l}(\mathrm{HCCH})_{2} \\
\text { T-Shaped }\end{array}$ & $\begin{array}{l}\mathrm{U}_{\text {dipole }} \\
\mathrm{C}_{3} / \mathrm{R}^{3}\end{array}$ & $\begin{array}{l}\mathrm{U}_{\text {dip-quad }} \\
\mathrm{C}_{4} / \mathrm{R}^{4}\end{array}$ & $\begin{array}{l}\mathrm{U}_{\text {quadrupole }} \\
\mathrm{C}_{5} / \mathrm{R}^{5}\end{array}$ & $\begin{array}{l}U_{\text {induction }} \\
-C_{6}^{\text {ind }} / R^{6}\end{array}$ & $\begin{array}{l}\mathrm{U}_{\text {dispersion }} \\
-\mathrm{C}_{6} / \mathrm{R}^{6}\end{array}$ & $\begin{array}{l}U_{\text {disp-C8 }} \\
-C_{8} / R^{8}\end{array}$ & $\begin{array}{l}\mathrm{U}_{\text {disp-c10 }} \\
-\mathrm{C}_{10} / \mathrm{R}^{10}\end{array}$ & $\mathrm{U}_{\text {Total }}$ & $\begin{array}{l}\text { MP4SDTQ/ } \\
6-311++\mathrm{G} \\
(3 \mathrm{df}, 3 \mathrm{pd})^{\mathrm{b}}\end{array}$ \\
\hline $\mathbf{A}^{\mathrm{d}}$ & 0.0 & 0.0 & -1.9456 & 0.0 & $\begin{array}{l}-0.3253 \\
(-0.3319)^{\mathrm{a}}\end{array}$ & -0.2273 & -0.08341 & -2.5816 & \\
\hline $\mathbf{B}^{\mathrm{e}}$ & 0.0 & 0.0 & -1.1923 & 0.0 & $\begin{array}{l}-0.3262 \\
(-0.3403)^{\mathrm{a}}\end{array}$ & -0.2256 & -0.08188 & -1.8260 & -1.32 \\
\hline $\begin{array}{l}(\mathrm{HCCH})_{2} \\
\text { Slipped-Parallel }\end{array}$ & $\begin{array}{l}\mathrm{U}_{\text {dipole }} \\
\mathrm{C}_{3} / \mathrm{R}^{3}\end{array}$ & $\begin{array}{l}\mathrm{U}_{\text {dip-quad }} \\
\mathrm{C}_{4} / \mathrm{R}^{4}\end{array}$ & $\begin{array}{l}\mathrm{U}_{\text {quadrupole }} \\
\mathrm{C}_{5} / \mathrm{R}^{5}\end{array}$ & $\begin{array}{l}\mathrm{U}_{\text {induction }} \\
-\mathrm{C}_{6}^{\text {ind }} / \mathrm{R}^{6}\end{array}$ & $\begin{array}{l}\mathrm{U}_{\text {dispersion }} \\
-\mathrm{C}_{6} / \mathrm{R}^{6}\end{array}$ & $\begin{array}{l}U_{\text {disp-C8 }} \\
-C_{8} / R^{8}\end{array}$ & $\begin{array}{l}\mathrm{U}_{\text {disp-C10 }} \\
-\mathrm{C}_{10} / \mathrm{R}^{10}\end{array}$ & $\mathrm{U}_{\text {Total }}$ & $\begin{array}{l}\text { MP4SDTQ/ } \\
6-311++\mathrm{G} \\
(3 \mathrm{df}, 3 \mathrm{pd}){ }^{\mathrm{b}}\end{array}$ \\
\hline $\mathrm{A}^{\mathrm{d}}$ & 0.0 & 0.0 & 2.8304 & 0.0 & $\begin{array}{l}-0.3926 \\
(-0.3993)^{a}\end{array}$ & -0.2921 & -0.1141 & 2.0316 & \\
\hline $\mathbf{B}^{\mathrm{e}}$ & 0.0 & 0.0 & 1.7345 & 0.0 & $\begin{array}{l}-0.3937 \\
(-0.3896)^{\mathrm{a}}\end{array}$ & -0.2898 & -0.1120 & 0.9390 & -1.21 \\
\hline $\begin{array}{l}\left(\mathrm{H}_{2}\right)_{2} \\
\text { T-Shaped } \\
b\end{array}$ & $\begin{array}{l}\mathrm{U}_{\text {dipole }} \\
\mathrm{C}_{3} / \mathrm{R}^{3}\end{array}$ & $\begin{array}{l}\mathrm{U}_{\text {dip-quad }} \\
\mathrm{C}_{4} / \mathrm{R}^{4}\end{array}$ & $\begin{array}{l}\mathrm{U}_{\text {quadrupole }} \\
\mathrm{C}_{5} / \mathrm{R}^{5}\end{array}$ & $\begin{array}{l}\mathrm{U}_{\text {induction }} \\
-\mathrm{C}_{6} \text { ind } / \mathrm{R}^{6}\end{array}$ & $\begin{array}{l}U_{\text {dispersion }} \\
-C_{6} / R^{6}\end{array}$ & $\begin{array}{l}\mathrm{U}_{\text {disp-C8 }} \\
-\mathrm{C}_{8} / \mathrm{R}^{8}\end{array}$ & $\begin{array}{l}\mathrm{U}_{\text {disp-C10 }} \\
-\mathrm{C}_{10} / \mathrm{R}^{10}\end{array}$ & $\mathrm{U}_{\text {Total }}$ & $\begin{array}{l}\text { MP4SDTQ/ } \\
\text { aug-cc-pV5Z }\end{array}$ \\
\hline $\begin{array}{l}\mathbf{A}^{\mathrm{d}} \\
\mathrm{B}^{\mathrm{e}}\end{array}$ & $\begin{array}{l}0.0 \\
0.0\end{array}$ & $\begin{array}{l}0.0 \\
0.0\end{array}$ & $\begin{array}{l}-0.03139 \\
-0.02768\end{array}$ & $\begin{array}{l}0.0 \\
0.0\end{array}$ & $\begin{array}{l}-0.08283 \\
-0.07929 \\
(0.08221)^{\mathrm{a}}\end{array}$ & $\begin{array}{l}-0.02794 \\
-0.02683\end{array}$ & $\begin{array}{l}-0.00495 \\
-0.00477\end{array}$ & $\begin{array}{l}-0.1471 \\
-0.1386\end{array}$ & -0.1047 \\
\hline $\begin{array}{l}\left(\mathrm{H}_{2}\right)_{2} \\
\text { Slipped-Parallel } \\
b\end{array}$ & $\begin{array}{l}\mathrm{U}_{\text {dipole }} \\
\mathrm{C}_{3} / \mathrm{R}^{3}\end{array}$ & $\begin{array}{l}\mathrm{U}_{\text {dip-quad }} \\
\mathrm{C}_{4} / \mathrm{R}^{4}\end{array}$ & $\begin{array}{l}\mathrm{U}_{\text {quadrupole }} \\
\mathrm{C}_{5} / \mathrm{R}^{5}\end{array}$ & $\begin{array}{l}\mathrm{U}_{\text {induction }} \\
-\mathrm{C}_{6}^{\text {ind }} / \mathrm{R}^{6}\end{array}$ & $\begin{array}{l}U_{\text {dispersion }} \\
-C_{6} / R^{6}\end{array}$ & $\begin{array}{l}U_{\text {disp-C8 }} \\
-C_{8} / R^{8}\end{array}$ & $\begin{array}{l}\mathrm{U}_{\text {disp-C10 }} \\
-\mathrm{C}_{10} / \mathrm{R}^{10}\end{array}$ & $\mathrm{U}_{\text {Total }}$ & $\begin{array}{l}\text { MP4SDTQ/ } \\
\text { aug-cc-pV5Z }\end{array}$ \\
\hline $\begin{array}{l}A^{d} \\
B^{e}\end{array}$ & $\begin{array}{l}0.0 \\
0.0\end{array}$ & $\begin{array}{l}0.0 \\
0.0\end{array}$ & $\begin{array}{l}0.03308 \\
0.02917\end{array}$ & $\begin{array}{l}0.0 \\
0.0\end{array}$ & $\begin{array}{l}-0.06906 \\
-0.06610 \\
(-0.06442)^{a}\end{array}$ & $\begin{array}{l}-0.02193 \\
-0.02105\end{array}$ & $\begin{array}{l}-0.00365 \\
-0.00352\end{array}$ & $\begin{array}{l}-0.0616 \\
-0.0615\end{array}$ & -0.0959 \\
\hline
\end{tabular}

a The dispersion energy evaluated taking into account a deviation from isotropicity of the polarizability (See equations (19) and (20)); ${ }^{\mathrm{b}}$ MP4(SDTQ) BSSE corrected values; ${ }^{\mathrm{c}}$ CCSD-T/aug-cc-pVQZ//MP2/aug-cc-pVTZ BSSE corrected value ${ }^{57} ;{ }^{\text {d }}$ In "A" available experimental electrical properties, given in Table 7, were utilized. For the water dimer the experimental geometry (See Table 1) was also used; ${ }^{\mathrm{e}}$ In "B" only $a b$ initio calculated values for the electrical properties, given in Table 7 , were used.

coefficients, $\mathrm{C}_{8}$ and $\mathrm{C}_{10}$, are also given in Table 6. Their absolute values are smaller than $\mathrm{C}_{6}$, and their behavior reflects that of a nicely converging power series, with the contribution of the next term, $-\mathrm{C}_{12} / \mathrm{R}^{12}$, expected to be practically negligible. However, the $\mathrm{C}_{8}$ and $\mathrm{C}_{10}$ coefficients make a visible contribution to the dispersion energy for all four dimers studied here, and can not be ignored.

It can be seen that for the $\left(\mathrm{H}_{2} \mathrm{O}\right)_{2}$ and $(\mathrm{HCN})_{2}$ dimers, the dipole and quadrupole electrostatic contributions accounts for $c a .60 \%$ of the interaction energy for the water dimer and $\mathrm{ca} .85 \%$ for the HCN dimer, for which the dispersion effects play a minor role. Comparing these results with the $a b$ initio calculations reported in Table 2 for the HCN dimer it can be seen that the difference between the MP4(SDTQ)/6-311++G(3df,3pd) BSSE corrected stabilization energy and the corresponding Hartree-Fock one is only $8 \%$ in total agreement with the long-range interaction energy calculations. In the case of the water 
Table 7. Electrical Properties for the isolated monomers: Dipole Moments $(\mu)$ in units of $10^{-30} \mathrm{Cm}$, Electric Quadrupole Moments $(\Theta)$ in units of $10^{-40} \mathrm{Cm}^{2}$, Electric Dipole Mean Static Polarizabilities $\left(\bar{\alpha}^{0}\right)$ in units of $10^{-40} \mathrm{C}^{2} \mathrm{~m}^{2} \mathrm{~J}^{-1}$, Ionization Energies $\left(\mathrm{E}_{\mathrm{A}}\right)$ in eV. $\alpha_{\perp}$ and $\alpha_{\|}$are the two independent components $\left(\bar{\alpha}=\frac{1}{3}\left(\alpha_{\|}+2 \alpha_{\perp}\right)\right)$

\begin{tabular}{|c|c|c|c|c|c|c|c|c|}
\hline & $\Theta_{\mathrm{zz}}{ }^{\mathrm{a}}$ & $\Theta_{y y}$ & $\Theta_{\mathrm{xx}}$ & $\bar{\alpha}^{0}$ & $\alpha_{\perp}$ & $\alpha_{\|}$ & $\mathrm{E}_{\mathrm{A}}$ & $\mu$ \\
\hline $\mathrm{H}_{2} \mathrm{O}:$ Theoretical $^{\mathrm{b}}$ & -0.34 & 8.82 & -8.48 & 1.58 & 1.55 & 1.65 & 13.856 & 6.36 \\
\hline $\mathrm{H}_{2} \mathrm{O}$ : Experimental ${ }^{\mathrm{c}}$ & $-0.43 \pm 0.10$ & $8.77 \pm 0.07$ & $-8.34 \pm 0.07$ & $1.65^{\mathrm{d}}$ & & & $12.607^{\mathrm{e}}$ & $6.17^{\mathrm{d}}$ \\
\hline $\mathrm{H}_{2}$ : Theoretical ${ }^{\mathrm{b}}$ & 2.07 & & & 0.854 & 0.756 & 1.050 & 16.218 & 0.0 \\
\hline $\mathrm{H}_{2}$ : Experimental ${ }^{\mathrm{f}}$ & 2.2 & & & $0.895^{\mathrm{c}} 0.879^{\mathrm{g}}$ & $0.790^{\mathrm{g}}$ & $1.035^{\mathrm{g}}$ & $15.427^{\mathrm{h}}$ & 0.0 \\
\hline HCCH: Theoretical b & 21.92 & & & 3.76 & 3.12 & 5.05 & 11.130 & 0.0 \\
\hline $\mathrm{HCCH}:$ Experimental ${ }^{\mathrm{c}}$ & 28 & & & $3.71 \mathrm{~g}$ & $2.70^{\mathrm{g}}$ & $5.70^{\mathrm{g}}$ & $11.4^{\mathrm{h}}$ & 0.0 \\
\hline HCN: Theoretical b & 7.41 & & & 2.76 & 2.32 & 3.66 & 13.410 & 10.06 \\
\hline $\mathrm{HCN}$ : Experimental ${ }^{\mathrm{c}}$ & $25.68^{\mathrm{I}}$ & & & $2.88^{g}$ & $2.14^{\mathrm{g}}$ & $4.36^{\mathrm{g}}$ & $13.8^{\mathrm{h}}$ & $9.41^{\mathrm{j}}$ \\
\hline
\end{tabular}

${ }^{\mathrm{a}}$ The $\mathrm{z}$ axis is the principal axis. For $\mathrm{H}_{2} \mathrm{O}$ the $\mathrm{z}$ axis is the $\mathrm{C}_{2 \mathrm{v}}$ symmetry axis. For linear molecules: $\Theta_{\mathrm{xx}}=\Theta_{\mathrm{yy}}=-1 / 2 \Theta_{\mathrm{zz}} ;{ }^{\mathrm{b}} \Theta, \mu$ and $\mathrm{E}_{\mathrm{A}}$ calculated at the MP2/6-311++G(3d,3p) level of theory and $\bar{\alpha}^{0}$ with the aug-cc-pVTZ basis set (MP2 calculation); ${ }^{2}$ See ref. ${ }^{46}$; ${ }^{\mathrm{dz}}$ See ref. ${ }^{72}$; ${ }^{\mathrm{e}}$ See ref. ${ }^{73}$; ${ }^{\mathrm{f}}$ See

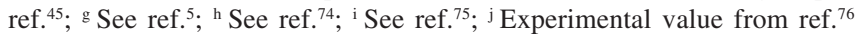

Table 8. Calculated Dispersion Coefficients: $\mathrm{C}_{6} / 10^{-79} \mathrm{Jm}^{6}\left(\right.$ or $10^{-60} \mathrm{ergcm}^{6}$ ), $\mathrm{C}_{8} / 10^{-99} \mathrm{Jm}^{8}$ (or $10^{-76} \mathrm{ergcm}^{8}$ ) and $\mathrm{C}_{10} / 10^{-119} \mathrm{Jm}^{10}\left(\right.$ or $10^{-92} \mathrm{ergcm}^{10}$ )

\begin{tabular}{|c|c|c|c|c|c|c|c|}
\hline & $\mathrm{C}_{6}^{\text {Expt. a }}$ & $\mathrm{C}_{6}^{A b \text {-initio b }}$ & $\mathrm{C}_{6}^{\text {asym. c }}$ & $\mathrm{C}_{8}$ Expt. a & $\mathrm{C}_{8}{ }^{A b-i n i t i o ~ b}$ & $\mathrm{C}_{10}$ Expt. a & $\mathrm{C}_{10}{ }^{\text {Ab-initio b }}$ \\
\hline$\left(\mathrm{H}_{2} \mathrm{O}\right)_{2}$ Trans-Linear & 33.32 & 33.57 & 15.38 & 216.29 & 229.38 & 737.20 & 822.76 \\
\hline$\left(\mathrm{HCN}_{2}^{2}\right.$ Linear & 111.10 & 100.10 & 46.79 & 1378.00 & 1165.60 & 8973.05 & 7125.63 \\
\hline$(\mathrm{HCCH})_{2} \mathrm{~T}-\mathrm{Shaped}$ & 152.30 & 152.72 & 159.31 & 2010.23 & 1994.48 & 13929.74 & 13674.49 \\
\hline$(\mathrm{HCCH})_{2}$ Slipped-Parallel & 152.30 & 152.72 & 151.15 & 2010.23 & 1994.48 & 13929.74 & 13674.49 \\
\hline$\left(\mathrm{H}_{2}\right)_{2} \mathrm{~T}-\mathrm{Shaped}$ & 11.99 & $\begin{array}{l}11.48 \\
{[11.56 \pm 0.32]^{\mathrm{d}}}\end{array}$ & 11.90 & 51.68 & 49.62 & 116.91 & 112.60 \\
\hline$\left(\mathrm{H}_{2}\right)_{2}$ Slipped-Parallel & 11.99 & 11.48 & 11.19 & 51.68 & 49.62 & 116.91 & 112.60 \\
\hline
\end{tabular}

a Evaluated using the experimental electrical properties given in Table 7; ${ }^{\mathrm{b}} \Theta, \mu$ and $\mathrm{E}_{\mathrm{A}}$ calculated at the MP2/6-311++G(3d,3p) level of theory and $\bar{\alpha}^{0}$ with the aug-cc-pVTZ basis set (MP2 calculation); ${ }^{c} \mathrm{C}_{6}{ }^{\text {asym }}$ calculated with equation (20); ${ }^{\mathrm{d}}$ Experimental value from Ref. ${ }^{7}$

dimer (see Table 1) the corresponding difference is $19 \%$, also in accordance with the results reported in Table 6. For the acetylene T-shaped dimer the dispersion contributions account for $35 \%$ of the interaction energy. However for the slipped-parallel structure the strongly repulsive quadrupole-quadrupole interaction contributions dominate the interaction energy, producing an unbound dimeric structure. Comparison with the $a b$ initio values reported in Table 3 for the $(\mathrm{HCCH})_{2}$ dimer reveal that the difference between the MP4 and HF values is $41 \%$ of the total interaction energy, in the line with the results reported in Table 6. Based on the analysis of Table 6 the $\left(\mathrm{H}_{2}\right)_{2}$ dimer is predominantly bound through dispersion forces. The quadrupole contribution for the T-shaped dimer correspond to only $20 \%$ of the interaction energy and for the slippedparallel this contribution is repulsive, similar to the acetylene dimer case, but in this case a bound structure is predicted due to the predominance of the dispersion effects. By comparison with the ab initio results from Table 4 , it can be seen that energy difference between the MP4(SDTQ) and HF calculations in this case is $86 \%$ of the MP4 interaction energy in nice agreement with the $80 \%$ contribution of the dispersion effected predicted by the multipole expansion approach. So, the importance of the induction-dispersion contributions to the total interaction energy can be placed in the following order, according to the long-range contributions to the interaction energy: $(\mathrm{HCN})_{2}<\left(\mathrm{H}_{2} \mathrm{O}\right)_{2}<(\mathrm{HCCH})_{2}<\left(\mathrm{H}_{2}\right)_{2}$. Comparing the total interaction energies reported in Table 6, with the MP4(SDTQ)/6-311++G(3df,3pd) BSSE corrected ab initio values quoted in the last column a very satisfactory agreement is found, showing that the multipole expansion approach can predict the energetic trend for these dimeric species, and therefore, can also be used to aid the understanding of the nature of the intermolecular forces in weakly bound complexes. The best agreement between the multipole expansion and Post-HF results is found when the calculated $a b$ initio electrical properties are used instead of the experimental tabulated values. It can be seen from Table 7 that in general the ab initio results are in good accordance with experiment, except for the electric quadrupole moments for the $\mathrm{HCN}$ and $\mathrm{HCCH}$ molecules. However, the overall agreement of the MP2/6$311++\mathrm{G}(3 \mathrm{~d}, 3 \mathrm{p})$ electric dipole and quadrupole moments and MP2/aug-cc-pVTZ electric dipole polarizabilities with experiment for a number of molecules is very satisfactory so, the use of these theoretical calculated electrical properties is recommended. 
We finally return to the discussion of the electron correlation effects in Table 9. There are basically two groups of methods for the calculation of the interaction energy $(\Delta \mathrm{E})$. Within the supermolecule approach $\left(\Delta \mathrm{E}^{\text {complex }}=\right.$ $\left.\mathrm{E}^{\text {complex }}-\Sigma\left(\mathrm{E}^{\text {monomers }}\right)\right)$, the interaction energy can be written as a sum of two contributions: the Hartree-Fock energy $\left(\Delta \mathrm{E}^{\mathrm{HF}}\right)$ and the correlation energy $\left(\Delta \mathrm{E}^{\mathrm{corr}}\right)$, where it is also assumed that both the isolated systems and the supersystem are described with sufficient accuracy. With the perturbation treatment, where the perturbation and the corresponding interactions energy must be sufficiently small, being therefore appropriate for studying weak interactions, the interaction energy is obtained directly as the sum of various energy contributions (Coulombic, induction, charge-transfer, exchange-repulsion, dispersion), so it is free of BSSE. A detailed discussion on this subject can be found for example in Chalasinski and Szczesniak ${ }^{77}$ and Jeziorski et al. $^{78}$ It can be shown that the supermolecule HF interaction energy includes all the above contributions, except for the dispersion energy. It can also be shown that the dispersion energy can be considered to be identical with the intersystem correlation energy for large distances. ${ }^{6,79}$
$\Delta \mathrm{E}=\Delta \mathrm{E}^{\mathrm{HF}}+\Delta \mathrm{E}^{\mathrm{corr}}$

In the supermolecule calculations using MPn Post-HF methods the correlation energy can be identified as the difference between the total MPn interaction energy and the HF contribution evaluated with the MPn fully optimized geometry, denominated here as $\Delta \mathrm{E}^{\text {corr }}$.

$\Delta \mathrm{E}^{\text {corr }}=\Delta \mathrm{E}_{\text {ele-nuc }}$ MPn $^{2}-\Delta \mathrm{E}_{\text {ele-nuc }}$ HF-contribution

Following the discussion from Hobza and Zahradník ${ }^{6}$ we can also write the correlation energy as the difference of the MPn stabilization energy and the HF energy, calculated with the fully optimized HF geometry $\left(\Delta \mathrm{E}^{\mathrm{corr}-\mathrm{HF}}\right)$.

$\Delta \mathrm{E}^{\text {corr-HF }}=\Delta \mathrm{E}_{\text {ele-nuc }}-\Delta \mathrm{E}_{\text {ele-nuc }} \mathrm{HF}$

The values represented by equations (23) and (24) are given in Table 9, along with the percentage contribution in relation to the total MPn interaction energy. This gives us a more realistic idea about the contribution of the HF energy. For reason of comparison the difference between

Table 9. Electronic correlation contributions to the dimer interaction energy. The percentage contribution of the correlation energy is given in parenthesis

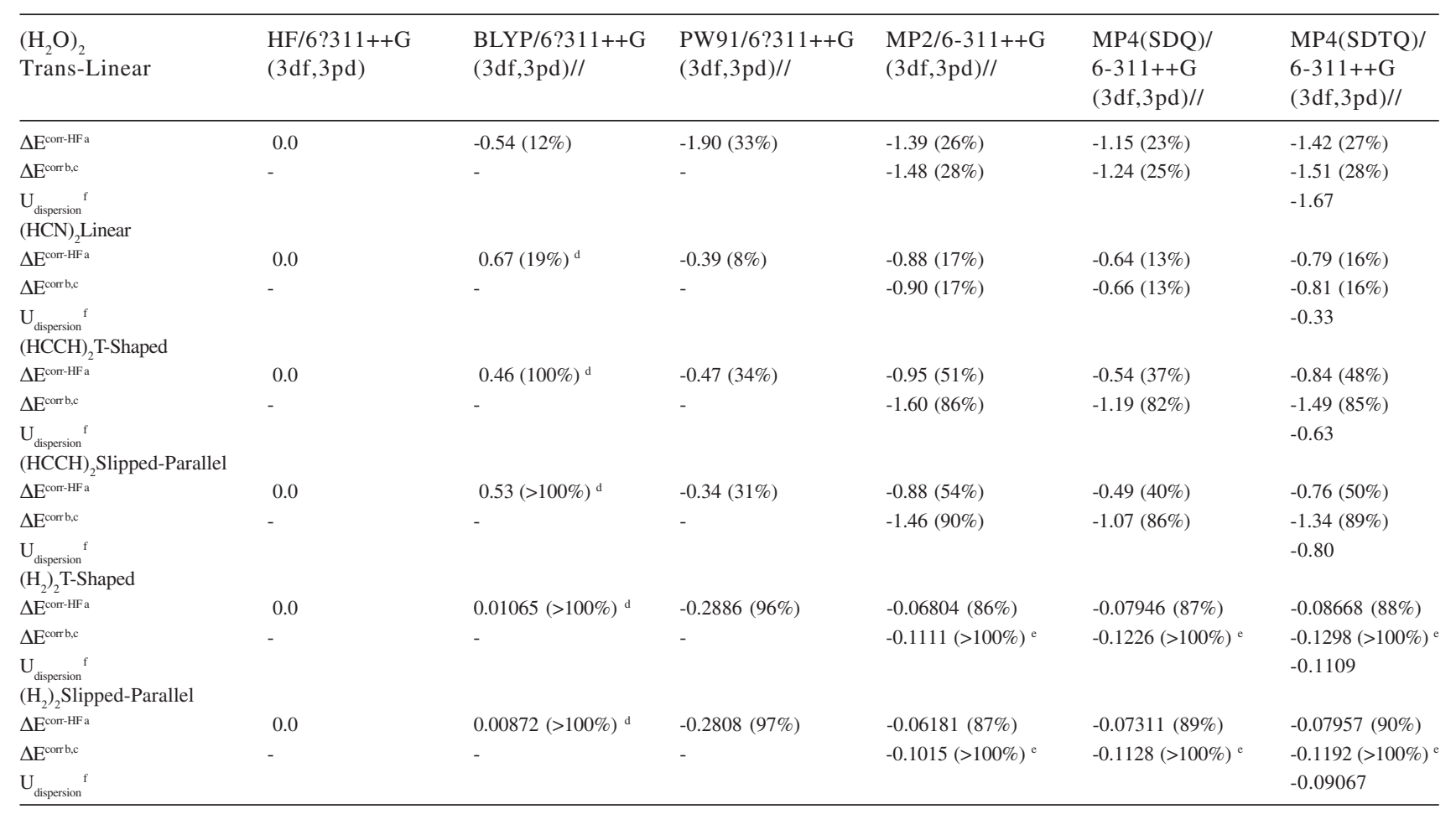

${ }^{\mathrm{a}} \Delta \mathrm{E}^{\text {corr-HF}}=\Delta \mathrm{E}_{\text {ele-nuc }}-\Delta \mathrm{E}_{\text {ele-nuc }}{ }_{\mathrm{HF} / 6-311++\mathrm{G}(3 \mathrm{df}, 3 \mathrm{pd})} ;{ }^{\mathrm{b}} \Delta \mathrm{E}^{\mathrm{corr}}=\Delta \mathrm{E}_{\text {ele-nuc }}{ }^{\mathrm{MPn}}-\Delta \mathrm{E}_{\text {ele-nuc }}{ }_{\mathrm{HF}-\text { contribution; }}^{\mathrm{c}}{ }^{\mathrm{B}}$ Evaluated using the Hartree-Fock (HF) contribution to the MP2 interaction energy $\left(\Delta \mathrm{E}^{\mathrm{MP} 2}=\Delta \mathrm{E}^{\mathrm{HF}}+\Delta \mathrm{E}^{(2)}\right)$; $^{\mathrm{d}}$ The correlation contribution is repulsive, in a sense that is increases the stabilization energy in relation

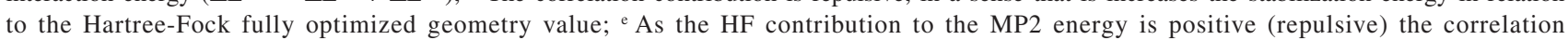
contribution is larger than the stabilization energy itself; ${ }^{\mathrm{f}} \mathrm{U}_{\text {dispersion }}=\mathrm{U}_{\text {disp-R6 }}+\mathrm{U}_{\text {disp-R8 }}+\mathrm{U}_{\text {disp-R10 }}$ (equations: (13), (15), (16)). 
the HF and DFT interaction energy (using the respective optimized geometries) is also given, and it may provide an assessment of the correlation energy contribution built in the exchange correlation functionals, where no partition of the correlation part is made as in the case of the ab initio Post-HF methods. It can be seen from Table 9 that for the water dimer the correlation contribution of the BLYP functional is $12 \%$, approximately half of the MP4(SDTQ) value $(27 \%)$, with the corresponding contribution for the PW91 functional being higher $(33 \%)$. For the $(\mathrm{HCN})_{2}$ dimer the BLYP correlation part is repulsive and the PW91 value is only $8 \%$, being this time half of the MP4(SDTQ) value $(16 \%)$. For the $(\mathrm{HCCH})_{2}$ and $\left(\mathrm{H}_{2}\right)_{2}$ dimers the BLYP dispersion contribution is systematically repulsive with the PW91 value being attractive, accounting for threequarters $(34 \%)$ of the MP4(SDTQ) value (48\%) for the acetylene dimer and $96 \%$ for the $\left(\mathrm{H}_{2}\right)_{2}$ dimer (larger than the MP4 value of $88 \%$ ). So, while a trend can be established for the BLYP functional, which appears to be unable to account for dispersion effects in weakly bound species, the behavior of the PW91 functional oscillates either underestimating or overestimating the correlation contribution, but, nevertheless, always yielding negative (attractive) values for the dispersion contribution. At this point, besides not having sufficient evidences, it is quite attempting to say that the PW91 functional has a tendency to produce somehow exaggerated attractive interactions, which may compensate the lack of dispersion effects present in the built in DFT exchange correlation functionals.

By looking back at Tables 1 to 4, it can be seen that the $\mathrm{HF}$ equilibrium intermolecular distance for the water and HCN dimers follow closely the MP2 optimized values, so $\Delta \mathrm{E}^{\mathrm{corr}}$ and $\Delta \mathrm{E}^{\mathrm{corr}-\mathrm{HF}}$ are practically the same and equations (22) and (23) can be used indiscriminately. In the case of the $(\mathrm{HCCH})_{2}$ and $\left(\mathrm{H}_{2}\right)_{2}$ weakly bound dimers the HF optimized intermolecular equilibrium distances are larger than the MP2 ones, producing considerably smaller interaction energies, and so, an enormous difference between $\Delta E^{\text {corr }}$ and $\Delta E^{\text {corr-HF }}$ is observed. For the $\left(\mathrm{H}_{2}\right)_{2}$ dimer the HF contribution to the MP2 interaction energy is even repulsive, therefore, the use of equation (23) for the definition of the correlation energy may not be wise. Nevertheless, the trend in the relative importance of the dispersion contribution can be clearly obtained using $\Delta \mathrm{E}^{\text {corr-HF}}$, equation (24), with respect to the MP4(SDTQ)/6$311++\mathrm{G}(3 \mathrm{df}, 3 \mathrm{pd})$ energy values: $(\mathrm{HCN})_{2} 16 \%,\left(\mathrm{H}_{2} \mathrm{O}\right)_{2} 27 \%$, $(\mathrm{HCCH})_{2} 48 \%,\left(\mathrm{H}_{2}\right)_{2} 88 \%$. This result is in accordance with the multipole expansion analysis reported in Table 6 , where according to the values obtained with the $a b$ initio calculated electrical properties for the isolated monomers (line B of Table 6), the following order for the dispersion contribution is found: $(\mathrm{HCN})_{2} 11 \%,\left(\mathrm{H}_{2} \mathrm{O}\right)_{2} 36 \%,(\mathrm{HCCH})_{2}$ $35 \%,\left(\mathrm{H}_{2}\right)_{2} 80 \%$. It can be seen that there is a good overall agreement between the dispersion long-range contributions to the interaction energy and the $a b$ initio MP4(SDTQ) correlation energy, $\Delta \mathrm{E}^{\mathrm{corr}-\mathrm{HF}}$ given by equation (24). The long-range dispersion energy $\left(\mathrm{U}_{\text {dispersion }}\right)$ is also quoted in Table 9 for reason of comparison. It can be seen that it approaches the correlation energy $\left(\Delta \mathrm{E}^{\mathrm{corr}-\mathrm{HF}}\right)$, as it has been discussed in details in ref. ${ }^{6}$ The results reported here definitively gives support for the utility of the multipole expansion long-range approach to help in the understanding of the nature of weak intermolecular interactions.

\section{Conclusion}

In this article four distinct dimers encompassing Linear, $\mathrm{C}_{2 \mathrm{v}}$-Trans-linear, T-Shaped and Slipped-Parallel equilibrium spatial arrangements were investigated using $a b$ initio HF and Post-HF (MP4(SDTQ), CCSD-T), DFT (BLYP and PW91 functionals) and Multipole Expansion methods, aiming to understand the role played by dispersion forces for the dimer stabilization. The electrostatic, induction and dispersion energy contributions to the long-range interaction energy were calculated, using the ab initio MP2/6-311++G(2d,2p) level of calculation for dimeric structural and monomer electrical properties calculations $(6-311++\mathrm{G}(3 \mathrm{~d}, 3 \mathrm{p})$ and aug-ccpVTZ basis sets). The $\mathrm{C}_{6}, \mathrm{C}_{8}, \mathrm{C}_{10}$ and asymmetric $\mathrm{C}_{6}\left(\mathrm{C}_{6}\right.$ asym $)$, which takes into account the deviation from the spherical symmetry, dispersion coefficients were calculated. The agreement with the $a b$ initio MP4(SDTQ)/6$311++\mathrm{G}(3 \mathrm{df}, 3 \mathrm{pd})$ correlation energy is fine, providing support for the use of the multipole expansion in the study of weakly bound complexes. The BSSE was accounted for using the counterpoise method. It was shown that the size of the BSSE correction may give an indication of how close to completeness a given basis set is. In this way, for the $\left(\mathrm{H}_{2}\right)_{2}$ dimer, the correlated consistent Dunning's augcc-pV5Z basis set yielded a BSSE correction value of less than $1 \%$ of the total interaction energy of $-0.106 \mathrm{kcal} \mathrm{mol}^{-1}$ (MP4(SDTQ) result), very likely to be within the experimental uncertainty for stabilization energy determination. An attempt to assess how much correlation effects is included in the BLYP and PW91 exchange correlation functionals was made. While the BLYP functional seems appropriate for describing water clusters, it provides a very poor description of the acetylene and $\left(\mathrm{H}_{2}\right)_{2}$ weakly bound dimers, therefore, being recommended only in situations where dispersion forces do not play an 
important role. Regarding the PW91 functional, it appears to carry a considerable amount of electron correlation effects, but the results reported here do not show a regular pattern. Therefore, in order to reach a definitive conclusive, a more detailed study is required.

\section{Acknowledgments}

The author would like to thank the Brazilian Agencies CNPq (Conselho Nacional de Desenvolvimento Científico e Tecnológico) and FAPEMIG (Fundação de Amparo à Pesquisa no Estado de Minas Gerais) for support.

\section{References}

1. Discussion of the Faraday Society $\mathbf{1 9 6 5}, 40$, issue dedicated to Intermolecular Forces.

2. Faraday Discussion of the Chemical Society 1982, 73, issue dedicated to van der Walls molecules.

3. Buckingham, A.D.; Fowler, P.W.; Hutson, J.M.; Chem. Rev. 1988, 88, 963.

4. Chem. Rev. 1994, 94, special issue on van der Waals molecules.

5. Hirschfelder, J.O.; Curtiss, C.F.; Bird, R.B.; Molecular Theory of Gases and Liquids, John Wiley \& Sons: New York, 1954.

6. Hobza, P.; Zahradník, R.; Studies in Physical and Theoretical Chemistry: Weak Intermolecular Interactions in Chemistry and Biology, Elsevier Scientific Publishing Co.: Amsterdam, 1980.

7. Maitland, G.C.; Rigby, M.; Smith, E.B.; Wakeham, W.A; Intermolecular Forces: Their Origin and Determination, Oxford University Press: Oxford, 1981.

8. Resende, S.M; De Almeida, W.B.; J. Chem. Phys. 1995, 102, 4184.

9. Resende, S.M; De Almeida, W.B.; Chem. Phys. 1996, 206, 1.

10. Resende, S.M.; Pliego Jr., J.R. ; De Almeida, W.B.; J. Braz. Chem. Soc. 1997, 8, 555.

11. Buckingham, A.D.; Faraday Discuss. 1965, No. 40, 232.

12. Hartree, D. R.; Proc. Camb. Phil. Soc. 1928, 24, 328.

13. Fock, V. A.; Z. Phys. 1930, 15, 126.

14. Levine, I.N.; Quantum Chemistry, Prentice Hall: New Jersey, 2000.

15. Parr, R.G.; Yang, W.; Density Functional Theory of Atoms and Molecules, Oxford University Press,: New York, 1989.

17. Becke, A.D.; Phys. Rev. A 1988, 38, 3098.

18. Lee, C.; Yang, W.; Parr, R.G.; Phys. Rev. B 1988, 37, 785.

19. Miehlich, B.; Savin, A.; Stoll, H.; Preuss, H.; Chem. Phys. Lett. 1989, 157, 200.

20. Burke, K.; Perdew, J.P.; Wang, Y. In Electronic Density Functional Theory: Recent Progress and New Directions; Dobson, J.F.; Vignale, G.;Das, M.P., eds., Plenum:New York, 1998.

21. Perdew, J.P. In Electronic Structure Of Solids '91; Ziesche, P.; Eschrig, H., eds., Akademie Verlag: Berlin, 1991, p. 11.
22. Perdew, J.P.; Chevary, J.A.; Vosko, S.H.; Jackson, K.A.; Pederson, M.R.; Singh, D.J.; Fiolhais, C.; Phys. Rev. B 1992, 46, 6671 .

23. Perdew, J.P.; Chevary, J.A.; Vosko, S.H.; Jackson, K.A.; Pederson, M.R.; Singh, D.J.; Fiolhais, C.; Phys. Rev. B 1993, 48, 4978.

24. Perdew, J.P.; Burke, K.; Wang, Y.; Phys. Rev. B 1996, 54, 16533.

25. Mfller, C.; Plesset, M.S.; Phys. Rev. 1934, 46, 618.

26. Szabo, A.; Ostlund, N.S.; Modern Quantum Chemistry, Introduction to Advanced Electronic Structure Theory, Dover Plublications, Inc.: New York, 1996.

27. Krishnan, R.; Binkley, J.S.; Seeger, R.; Pople, J.A.; J. Chem. Phys. 1980, 72, 650.

28. McLean, A.D.; Chandler, G.S.; J. Chem. Phys. 1980, 72, 5639.

29. Clark, T.; Chandrasekhar, J.; Spitznagel, G.W.; Schleyer, P.v.R.; J. Comp. Chem. 1983, 4, 294.

30. Frisch, M.J.; Pople, J.A.; Binkley, J.S.; J. Chem. Phys. 1984, 80, 3265.

31. Binning Jr., R.C.; Curtiss, L.A.; J. Comput. Chem. 1990, 11, 1206.

32. McGrath, M.P.; Radom, L.; J. Chem. Phys. 1991, 94, 511.

33. Curtiss, L.A.; McGrath, M.P. ; Blandeau, J.-P.; Davis, N.E.; Binning Jr., R.C.; Radom, L.; J. Chem. Phys. 1995, 103, 6104.

34. Schlegel, H.B.; Frisch, M.J.; Int. J. Quantum Chem. 1995, 54, 83.

35. Woon, D.E.; Dunning Jr., T.H.; J. Chem. Phys. 1993, 98, 1358.

36. Kendall, R.A.; Dunning Jr., T.H.; Harrison, R.J.; J. Chem. Phys. 1992, 96, 6796.

37. Dunning Jr., T.H.; J. Chem. Phys. 1989, 90, 1007.

38. Peterson, K.A.; Woon, D.E.; Dunning Jr., T.H.; J. Chem. Phys. 1994, 100, 7410 .

39. Wilson, A.; van Mourik, T.; Dunning Jr., T.H.; J. Mol. Struct. (THEOCHEM) 1997, 388, 339.

40. Davidson, E.R.; Chem. Phys. Lett. 1996, 220, 514.

41. Jansen, H.B.; Ros, P.; Chem. Phys. Lett. 1969, 3,140.

42. Boys, S.F.; Bernardi, F.; Molec. Phys. 1970, 19, 553.

43. van Duijneveldt, F.B.; van Duijneveldt-van de Rigdt, J.G.C.M.; van Lenthe, J.H.; Chem. Rev. 1994, 94, 1873.

44. Resende, S.M.; De Almeida, W.B.; van Duijneveldt van de Rijdt, J.G.C.M.; van Duijneveldt, F.B.; J. Chem. Phys. 2001, 115,2476

45. Hinchliffe, A.; Munn, R.W.; Molecular Eletromagnetism, John Wiley \& Sons: Chichester, 1985.

46. Hinchliffe, A.; Ab Initio Determination of Molecular Properties, Adam Hilger: Bristol, 1987.

47. London, F.; Z. Phys. Chem. 1930, B11, 222.

48. Margenau, H.; J. Chem. Phys. 1938, 9, 896.

49. Hornig, J.F.; Hirschfelder, J.O.; J. Chem. Phys. 1952, 20, 1812. 
50. Frisch, M. J.; Trucks, G. W.; Schlegel, H. B.; Scuseria, G. E.; Robb, M. A.; Cheeseman, J. R.; Zakrzewski, V. G.; Montgomery, Jr., J. A ; Stratmann, R. E.; Burant, J. C.; Dapprich, S.;. Millam, J. M; Daniels, A. D.; Kudin, K. N.; Strain, M. C.; Farkas, O.; Tomasi, J.; Barone, V.; Cossi, M.; Cammi, R.; Mennucci, B.; Pomelli, C.; Adamo, C.; Clifford, S.; Ochterski, J.; Petersson, G. A.; Ayala, P. Y.; Cui, Q.; Morokuma, K.; Malick, D. K.; Rabuck, A. D.; Raghavachari, K.; Foresman, J. B.; Cioslowski, J.; Ortiz, J. V.; Stefanov, B. B.; Liu, G.; Liashenko, A.; Piskorz, P.; Komaromi, I.; Gomperts, R.; Martin, R. L.; Fox, D. J.; Keith, T.; Al-Laham, M. A.; Peng, C. Y.; Nanayakkara, A.; Gonzalez, C.; Challacombe, M. ; Gill, P. M. W.; Johnson, B. ; Chen, W.; Wong, M. W.; Andres, J. L.; Gonzalez, C.; Head-Gordon, M.; Replogle, E. S.; Pople, J. A; Gaussian 98, Revision A.6, Gaussian, Inc.: Pittsburgh PA, 1998.

51. De Abreu, H.A.; Ph.D. Thesis, Universidade Federal de Minas Gerais, Belo Horizonte, 2004.

52. Nascimento Jr., C.S.; Dos Santos, H.F.; De Almeida, W.B.; Chem. Phys. Lett. 2004, 397, 422.

53. Nascimento, Jr., C.S.; Anconi, C.P.A.; Dos Santos, H.F.; De Almeida, W.B.; J. Phys. Chem. A 2005, 109, 3209.

54. Dyke, T.R.; Muenter, J.S.; J. Chem.Phys. 1974, 60, 2929.

55. Dyke, T.R.; Mack, K.M.; Muenter, J.S.; J. Chem.Phys. 1977, 66, 498.

56. Bartlett, R.J.; Stanton, J.F.; In Reviews in Computational Chemistry, Aplications of Post-Hartree-Fock Methods; Lipkowitz, K.B.; Boyd, D.B., eds., VCH Publishers, Inc: New York, 1994, vol. 5, ch. 3.

57. De Almeida, W.B.; Memoirs of Full Professorship Appointment, Universidade Federal de Minas Gerais, Belo Horizonte, 2004.

58. Dyke, T.R.; Muenter, J.S.; J. Chem.Phys. 1972, 57, 5011.

59. Legon, A.C.; Millen, D.J.; Faraday Discuss. 1982, 73, 128.

60. Buxton, L.W.; Campbell, E.J.; Flygare, W.H.; Chem. Phys. 1981, 56, 399.
61. Fraser, G.T.; Suenram, R.D.; Lovas, F.J.; Pine, A.S.; Hougen, J.T; Lafferty, W.J.; Muenter, J.S.; J. Chem. Phys. 1988, 89, 6028.

62. De Almeida, W.B.; J. Braz. Chem. Soc. 1997, 8, 77.

63. Prichard, D.G.; Nandi, R.N.; Muenter, J.S.; J. Chem. Phys. 1989, 89, 115.

64. Ohshima, Y.; Matsumoto, Y.; Takami, M.; Kuchitsu, K.; Chem. Phys. Lett. 1988, 147, 1.

65. Fraser, G.T.; J. Chem. Phys. 1989, 90, 2097.

66. McKellar, A.R.W.; Welsh, H.L.; Can. J. Phys. 1974, 52, 1082.

67. Gordon, R.G.; Cashion, J.K.; J. Chem. Phys. 1966, 44, 1190.

68. Michels, A.; Goudeket, M.; Physica 1941, 8, 347.

69. Mason, E.A.; Rice, W.E.; J. Chem. Phys. 1954, $22,522$.

70. Farrar, J.M.; Lee, Y.T.; J. Chem. Phys. 1972, 57, 5492.

71. Lennard-Jones, J.E.; Proc. Roy. Soc. 1924, A106, pp. 441, 443.

72. Atkins, P.W.; Physical Chemistry, $4^{\text {th }}$ ed.; Oxford University Press: Oxford, 1990.

73. McQuarrie, D.A.; Simon, J.D.; Physical Chemistry, A Molecular Approach, University Science Books: Sausalito, CA, 1997.

74. Weast, R.C.; Astle, M.J., eds.; Handbook of Chemistry and Physics, CRC Press, Inc.: Boca Raton, FL, 1981.

75. Townes, C.H.; Schawlow, A.L.; Microwave Spectroscopy, Dover Publications Inc.: New York, 1975.

76. Hinchliffe, A.; Computational Quantum Chemistry, Wiley: New York, 1988.

77. Chalasinski, G.; Szczesniak, M.M.; Chem. Rev. 1994, 94, 1723.

78. Jeziorski, B.; Moszynski, R.; Szalewicz, K.; Chem. Rev. 1994, 94, 1887.

79. See also: Hobza, P.; Zahradník, R.; Chem. Rev. 1988, 88, 871.

Received: November 29, 2004 Published on the web: April 26, 2005 